\title{
Mechanistic Origin of Superionic Lithium Diffusion in Anion-Disordered $\operatorname{Li}_{6} \mathbf{P S}_{5} X$ Argyrodites
}

\author{
Benjamin J. Morgan*1,2 \\ ${ }^{1}$ Department of Chemistry, University of Bath, Claverton Down BA2 7AY, United Kingdom \\ ${ }^{2}$ The Faraday Institution, Quad One, Harwell Science and Innovation Campus, Didcot OX11 ORA, United Kingdom*
}

(Dated: December 18, 2020)

\begin{abstract}
The rational development of fast-ion-conducting solid electrolytes for all-solid-state lithium-ion batteries requires understanding the key structural and chemical principles that give some materials their exceptional ionic conductivities. For the lithium argyrodites $\mathrm{Li}_{6} \mathrm{PS}_{5} X(X=\mathrm{Cl}, \mathrm{Br}, \mathrm{I})$, the choice of the halide, $X$, strongly affects the ionic conductivity, giving room-temperature ionic conductivities for $X=\{\mathrm{Cl}, \mathrm{Br}\}$ that are $\times 10^{3}$ higher than for $X=\mathrm{I}$. This variation has been attributed to differing degrees of $\mathrm{S} / X$ anion disorder. For $X=\{\mathrm{Cl}, \mathrm{Br}\}$ the $\mathrm{S} / X$ anions are substitutionally disordered, while for $X=\mathrm{I}$ the anion substructure is fully ordered. To better understand the role of substitutional anion disorder in enabling fast lithium-ion transport, we have performed a first-principles molecular dynamics study of $\mathrm{Li}_{6} \mathrm{PS}_{5} \mathrm{I}$ and $\mathrm{Li}_{6} \mathrm{PS}_{5} \mathrm{Cl}$ with varying amounts of $\mathrm{S} / X$ anion-site disorder. By considering the $\mathrm{S} / X$ anions as a tetrahedrally close-packed substructure, we identify three partially occupied lithium sites that define a contiguous three-dimensional network of face-sharing tetrahedra. The active lithium-ion diffusion pathways within this network are found to depend on the $\mathrm{S} / X$ anion configuration. For anion-disordered systems, the active site-site pathways give a percolating three-dimensional diffusion network; whereas for anion-ordered systems, critical site-site pathways are inactive, giving a disconnected diffusion network with lithium motion restricted to local orbits around $\mathrm{S}$ positions. Analysis of the lithium substructure and dynamics in terms of the lithium coordination around each sulfur site highlights a mechanistic link between substitutional anion disorder and lithium disorder. In anion-ordered systems the lithium ions are pseudo-ordered, with preferential 6-fold coordination of sulfur sites. Long-ranged lithium diffusion would disrupt this $\mathrm{SLi}_{6}$ pseudoordering, and is therefore disfavoured. In anion-disordered systems, the pseudo-ordered 6-fold S-Li coordination is frustrated due to $\mathrm{Li}-\mathrm{Li}$ Coulombic repulsion. Lithium positions become disordered, giving a range of $\mathrm{S}-\mathrm{Li}$ coordination environments. Long-ranged lithium diffusion is now possible with no net change in S-Li coordination numbers. This gives rise to superionic lithium transport in the anion-disordered systems, effected by a concerted string-like diffusion mechanism.
\end{abstract}

\section{INTRODUCTION}

Lithium-ion-conducting solid electrolytes are considered candidate materials for use in future all-solidstate lithium-ion batteries [1-3]. Present-day commercial lithium-ion batteries use liquid-organic electrolytes; these are flammable, raising safety issues, and have narrow electrochemical stability windows, preventing their use with energy-dense high-voltage electrodes. One possible solution is to instead use solid electrolytes, which ideally should be electrochemically inert, mechanically robust, have negligible electronic transport, and have high lithium-ion conductivities [4].

Although a number of highly conducting solid lithiumion electrolytes are known, none meet all the criteria for general commercial use [1,4-6]. Identifying new solid lithium-ion electrolytes is an active area of research [3], with strategies ranging from targeted chemical modification of known solid electrolytes, to improve their conductivities [7-11], to high-throughput screening of new materials [12-15]. In both cases, it is useful to understand why some materials are highly-conducting, yet others are not [3, 16-20]. Such understanding can help inform chemical strategies for optimising the ionic conductivities of known materials, or can provide selection criteria for identify-

\footnotetext{
* b.j.morgan@bath.ac.uk
}

ing new promising electrolytes. Particular insight can be gained from studying families of solid electrolytes that are superficially similar - such as those that share a common structural motif - but that exhibit quite different ionic conductivities $[2,19]$, as this can help reveal the fundamental mechanisms and key material characteristics that govern fast-ion conduction.

One family of promising lithium-ion solid-electrolytes are the lithium argyrodites $\mathrm{Li}_{6} \mathrm{PS}_{5} X(X=\mathrm{Cl}, \mathrm{Br}$, I) $[11,21-25]$. While $\mathrm{Li}_{6} \mathrm{PS}_{5} \mathrm{Cl}$ and $\mathrm{Li}_{6} \mathrm{PS}_{5} \mathrm{Br}$ exhibit high room-temperature ionic conductivities $\left(\sigma_{\mathrm{RT}} \approx\right.$ $\left.10^{-3} \mathrm{~S} \mathrm{~cm}^{-1}\right), \mathrm{Li}_{6} \mathrm{PS}_{5} \mathrm{I}$ is considerably less conductive $\left(\sigma_{\mathrm{RT}} \approx 10^{-6} \mathrm{~S} \mathrm{~cm}^{-1}\right)[26,27]$. The large difference between $X=\{\mathrm{Cl}, \mathrm{Br}\}$ and $X=\mathrm{I}$ is notable because these three materials have topologically identical crystal structures, suggesting the same lithium-ion diffusion pathways should exist in each system. This inverse correlation between anion size and ionic conductivity also runs counter to the trend seen in other families of solid electrolytes, for example, thio-LISICON and NASICON, in which larger, more polarisable, less electronegative anions are associated with increased ionic conductivities [2] — with this relationship often attributed to a combination of larger anions giving an increased accessible volume for the diffusing lithium ions and weaker lithium-anion electrostatic interactions.

A partial explanation for the ionic conductivity trend in the $\mathrm{Li}_{6} \mathrm{PS}_{5} X$ argyrodites comes from the observation 
that in these materials high conductivities are correlated with substitutional $\mathrm{S} / X$ anion disorder [23, 27]. In $\mathrm{Li}_{6} \mathrm{PS}_{5} \mathrm{I}$ the anions are fully ordered, and $\mathrm{S}$ and $\mathrm{I}$ atoms each fully occupy crystallographically distinct $4 c$ and $4 a$ Wyckoff positions, respectively. In $\mathrm{Li}_{6} \mathrm{PS}_{5} \mathrm{Cl}$ and $\mathrm{Li}_{6} \mathrm{PS}_{5} \mathrm{Br}$ the $\mathrm{S}$ and $\mathrm{Cl}$, or $\mathrm{S}$ and $\mathrm{Br}$, atoms are substitutionally disordered, which has been attributed to their similar ionic radii $[11,24,28]$ giving a low formation energy for $\mathrm{S} / X$ antisites [29, 30]. Molecular dynamics simulations of $\mathrm{Li}_{6} \mathrm{PS}_{5} X$ in which the degree of $\mathrm{S} / X$ disorder has been systematically varied provide additional evidence for a causal link between anion substitutional disorder and fast lithium-ion transport [22, 30-34].

Simulations performed on $\mathrm{Li}_{6} \mathrm{PS}_{5} X$ models with fullyordered $\mathrm{S} / X$ atoms predict low lithium diffusion coefficients and highly localised lithium motion, with lithium ions restricted to discrete "cages" surrounding the S atoms. In contrast, simulations performed on $\mathrm{S} / X$ disordered models predict high lithium diffusion coefficients, with lithium ions moving through a contiguous three-dimensional diffusion network. Despite this experimental and computational evidence linking lithium-ion conductivities in $\mathrm{Li}_{6} \mathrm{PS}_{5} X$ argyrodites with the degree of $\mathrm{S} / X$ disorder, a mechanistic model that explains this relationship is currently lacking.

To address this question, we have performed a firstprinciples molecular dynamics study of $\mathrm{Li}_{6} \mathrm{PS}_{5} \mathrm{I}$ and $\mathrm{Li}_{6} \mathrm{PS}_{5} \mathrm{Cl}$ with varying amounts of $\mathrm{S} / X$ anion-site disorder. We find that the lithium substructure can be generally described in terms of partial occupation of three crystallographically distinct tetrahedral sites that define a contiguous three-dimensional network. The pattern of active and inactive lithium-ion diffusion paths within this network, however, depends on the degree of $\mathrm{S} / X$ disorder. In anion-ordered systems, lithium site positions are displaced towards neighbouring sulfur sites due to electrostatic S-Li attraction, giving an ordered pattern of "inactive" site-site paths. In anion-disordered systems, however, the lithium site positions are statically disordered, and the set of active site-site paths forms a percolating three-dimensional network that permits longranged lithium diffusion.

We also have analysed our simulation trajectories by considering clusters of lithium ions as "coordination polyhedra" located around S anions. This perspective provides insight into the spatial correlations and collective dynamics in these groups of lithium ions. In the anionordered systems, the lithium-ions are pseudo-ordered, and preferentially form 6-coordinate polyhedra around sulfur atoms. While lithium movement within these $\mathrm{SLi}_{6}$ units is frequent, lithium exchange between $\mathrm{SLi}_{6}$ units is rare on a simulation timescale. We explain this by considering lithium exchange as a form of "defect formation", which is energetically disfavoured. In the aniondisordered systems, however, strong Coulombic interactions between nearby lithium ions frustrate the otherwise preferable 6-fold $\mathrm{S}-\mathrm{Li}$ coordination, producing a range of disordered $\mathrm{SLi}_{x}(x \geq 6)$ coordination environments.

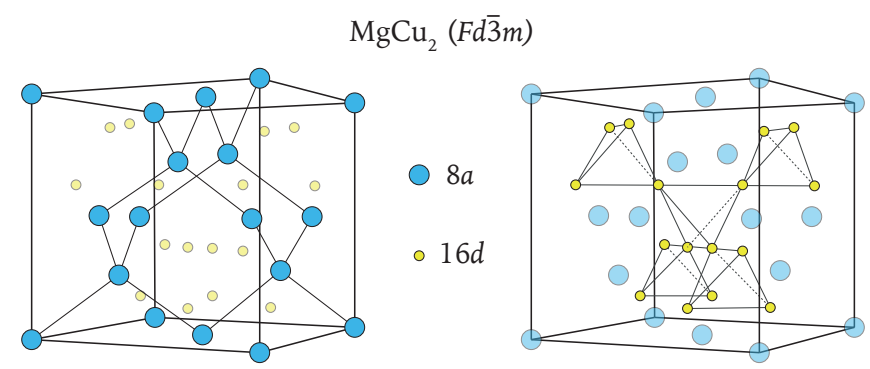

Argyrodite aristotype $(F \overline{4} 3 m)$

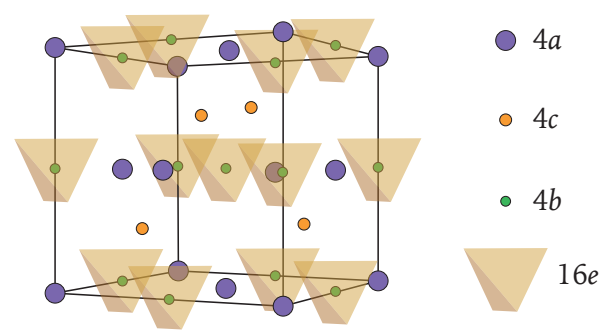

FIG. 1. Top panel: The $\mathrm{MgCu}_{2}$ structure with $\mathrm{Mg}$ (blue) occupying $8 a$ sites and $\mathrm{Cu}$ (yellow) occupying $16 d$ sites. The centre of each $16 d$ tetrahedra is a vacant $8 b$ site. Bottom panel: The cubic argyrodite aristotype. Half of the $F d \overline{3} m$ $8 b$ sites are occupied by $\mathrm{P}$, becoming $4 b$ sites in the reducedsymmetry $F \overline{4} 3 m$ space group, while the $F d \overline{3} m 8 a$ sites are split into symmetry inequivalent $F \overline{4} 3 m 4 a$ and $4 c$ sites [35, 36].

Lithium movement between coordination polyhedra is now possible without a net change in $\mathrm{S}-\mathrm{Li}$ coordination, making long-ranged lithium diffusion a viable lowenergy process. Further analysis of the dynamical correlations between mobile lithium ions reveals a concerted string-like "superionic" diffusion mechanism in the aniondisordered argyrodites. These results provide a mechanistic explanation for the exceptional ionic conductivities of anion-disordered $\mathrm{Li}_{6} \mathrm{PS}_{5} X$ argyrodites, and show how configurational framework disorder in solid electrolytes can cause static disorder amongst mobile ions, which consequently facilitates superionic conductivity.

\section{STRUCTURAL CONSIDERATIONS}

The $\mathrm{Li}_{6} \mathrm{PS}_{5} X$ argyrodites typically adopt a cationdisordered cubic aristotype in the $F \overline{4} 3 m$ space group, which can be considered to be derived from the $\mathrm{MgCu}_{2}$ cubic Laves phase ( $F d \overline{3} m$ space group) [21, 35, 37]. In $\mathrm{MgCu}_{2}$, the $\mathrm{Mg}$ sites (8a) form a diamond-structured array, and the $\mathrm{Cu}$ sites (16d) form an interpenetrating corner-sharing network of tetrahedra (Fig. 1, upper panel). In $\mathrm{Li}_{6} \mathrm{PS}_{5} X$, the phosphorus atoms occupy only half of the "Cu" tetrahedra, reducing the crystal symmetry from $F d \overline{3} m$ to $F \overline{4} 3 m$. The "Cu" sites (now denoted $16 e$ ) are fully occupied by $\mathrm{S}$, forming a face-centered cu- 
bic array of $\mathrm{PS}_{4}$ tetrahedra, and the "Mg" sites (now split into $4 a$ and $4 c$ ) are occupied by an equal ratio of $\mathrm{S}$ and $X$ anions (Fig. 1, lower panel) [35, 36]. In $\operatorname{Li}_{6} \mathrm{PS}_{5} \mathrm{I}$ the anions are ordered, with I atoms fully occupying the $4 a$ sites and $\mathrm{S}$ atoms fully occupying the $4 c$ sites. In $\mathrm{Li}_{6} \mathrm{PS}_{5} \mathrm{Cl}$ and $\mathrm{Li}_{6} \mathrm{PS}_{6} \mathrm{Br}$ the $\mathrm{Cl} / \mathrm{Br}$ and $\mathrm{S}$ atoms are disordered, with both anions distributed over the $4 a$ and the $4 c$ sites.

This three-dimensional arrangement of anions at $4 a$, $4 c$, and $16 e$ positions defines a tetrahedrally close-packed lattice [37-39]. The centres of these tetrahedra represent interstitial sites available to accommodate immobile cations (such as $\mathrm{P}$ ) or mobile lithium ions. In $\mathrm{MgCu}_{2}$ the $\mathrm{Mg}$ and $\mathrm{Cu}$ positions define the vertices of three crystallographically distinct tetrahedral sites. The lower crystal symmetry of the argyrodites splits these into six distinct tetrahedral types, which are listed in Table I, and were first described by Deiseroth et al. [36]. $\mathrm{In}_{\mathrm{Li}_{6}} \mathrm{PS}_{5} X$, one set of tetrahedra (type 0 ) is occupied by phosphorus, while the remaining tetrahedra (types $1-5$ ) are available to potentially accommodate lithium. The type 3 tetrahedra are centred on the $4 d$ Wyckoff positions with four $16 e$ sites as vertices, which they share with the type 0 $\mathrm{PS}_{4}$ tetrahedra. The remaining tetrahedra types 1,2 , 4 , and 5 form face-sharing cages around the $4 a$ and $4 c$ $\mathrm{S} / X$ sites. These cages each contain 28 tetrahedra, and each tetrahedron represents one lithium interstitial site (Fig. 2). The $4 a$ and $4 c$ coordination polyhedra are topologically identical: each has twelve pentagonal faces and four hexagonal faces, with the face-centres forming a 16vertex Frank-Kasper polyhedron [35, 40]. The hexagonal faces of these coordination polyhedra are arranged tetrahedrally around each central $\mathrm{S} / X$ site and are comprised of alternating type 2 and type 5 tetrahedral sites, which are shared between adjacent $4 a$ and $4 c$-coordination polyhedra.

In high-temperature modifications of $\mathrm{Li}_{6} \mathrm{PS}_{5} X$, lithium is disordered over the available tetrahedral sites types 1-5. X-ray single-crystal data for hightemperature- $\mathrm{Li}_{6} \mathrm{PS}_{5} \mathrm{I}$ show that electron density associated with these disordered lithium ions is smeared out over an extended region, but is predominantly associated with type 5 tetrahedra $[21,36]$. Subsequent neutron diffraction studies have typically assigned $\mathrm{Li}$ in $\mathrm{Li}_{6} \mathrm{PS}_{5} X$ as primarily occupying either $48 h$ sites - located within the type 5 tetrahedra - or $24 g$ sites - located at the shared face between adjacent type 5 tetrahedral pairs $[23,27,41]$, and denoted as type 5 a by Deiseroth et al. [36]. The standard model for lithium diffusion in $\mathrm{Li}_{6} \mathrm{PS}_{5} X$ considers only these type $548 h$ and type $5 \mathrm{a} 24 \mathrm{~g}$ positions, with microscopic lithium motion assumed to occur as a sequence of stochastic "jumps" between these sites $[23,28,30,32,42-44]$. Because type 5 tetrahedra form disconnected face-sharing pairs, a description of lithium transport that only considers the type 5 and type $5 \mathrm{a}$ sites is necessarily incomplete: any lithium motion beyond simple hopping back-and-forth within paired type 5 sites must involve other tetrahedral site
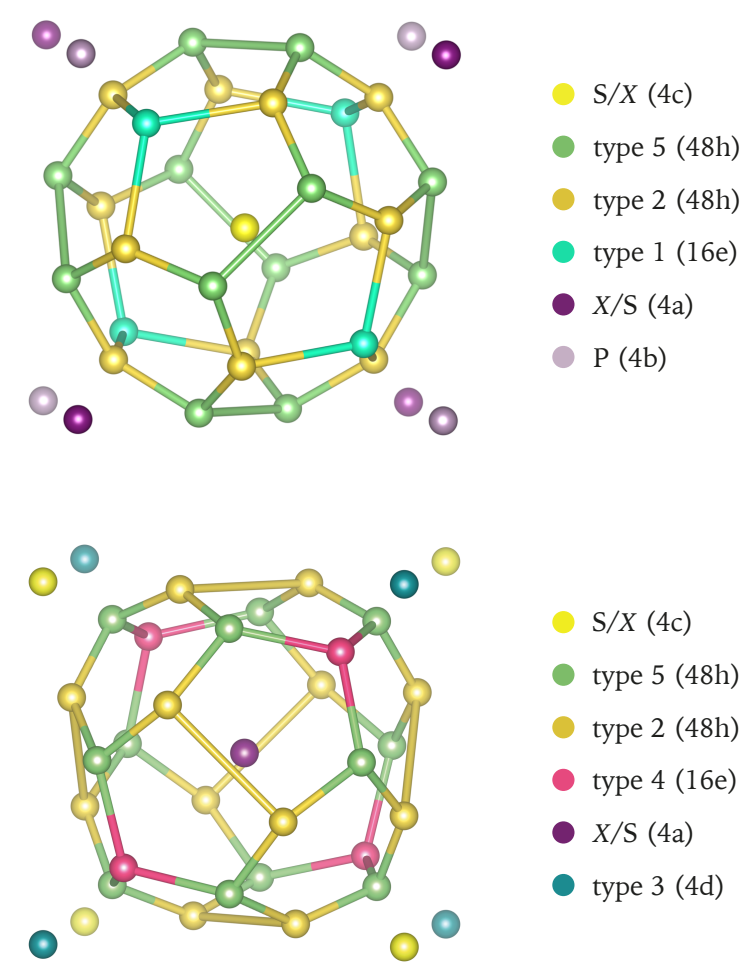

FIG. 2. (Top) The centres of the 28 tetrahedral sites surrounding each $4 c$ position. These define a truncated triakis tetrahedron [40], centered inside a cube of neighbouring $4 a$ and $4 c$ sites. (Bottom) Each $4 a$ site is coordinated by a topologically identical set of sites, centered inside a cube of $4 a$ and $4 b$ sites. The lithium-site coordination polyhedra around neighbouring $4 a$ and $4 c$ sites are linked by shared hexagonal faces consisting of type 2 and type 5 positions.

types [45].

The capacity for non-type 5 tetrahedra to accommodate lithium may therefore determine the degree to which lithium can diffuse through the structure. Some computational evidence for the role of non-type 5 tetrahedra in lithium diffusion in $\mathrm{Li}_{6} \mathrm{PS}_{5} X$ argyrodites comes from previous bond-valence calculations, which predict three distinct lithium sites [26, 27, 29]. Non-type 5 sites have also been identified in recent neutron diffraction studies of $\mathrm{Li}_{6} \mathrm{PS}_{5} \mathrm{Br}$ and $\mathrm{Li}_{6} \mathrm{PS}_{5} \mathrm{Cl}[46,47]$, as well as in lithiumargyrodites with lithium stoichiometries $x(\mathrm{Li})>6[48-$ 50]. A general mechanistic description of lithium conduction in lithium-argyrodites that describes the role of different lithium sites and that can explain the relationship between substitutional anion disorder and fast lithium transport, however, is currently lacking.

\section{METHODS}

To simulate lithium dynamics in $\mathrm{Li}_{6} \mathrm{PS}_{5} \mathrm{I}$ and $\mathrm{Li}_{6} \mathrm{PS}_{5} \mathrm{Cl}$, we have performed a series of ab initio molecular dynamics simulations using VASP [51, 52]. For 


\begin{tabular}{lll}
\hline $\begin{array}{l}\text { Type } \\
\text { tetrahedron centre }\end{array}$ & \\
\hline 0 & $4 b(\mathrm{P})$ & Centre of $\mathrm{PS}_{4}$ tetrahedra. \\
1 & $16 e$ & 4 -fold coordination of $4 c$ sites. \\
2 & $48 h$ & 12 -fold coordination of $4 a$ and $4 c$ sites. Form face-sharing pairs around $4 a$ sites. \\
3 & $4 d$ & Four common corners with neighbouring $\mathrm{PS}_{4}$ tetrahedra. \\
4 & $16 e$ & 4 -fold coordination of $4 a$ sites. \\
5 & $48 h$ & 12 -fold coordination of $4 c$ and $4 a$ sites. Form face-sharing pairs around $4 c$ sites. \\
\hline
\end{tabular}

TABLE I. Tetrahedral holes formed by the close-packed $\mathrm{S} / X$ anion substructure in $\operatorname{Li}_{6} \mathrm{PS}_{5} X$ ( $F \overline{4} 3 m$, setting 2$)$, following the classification of Deiseroth et al. [36].

all calculations we have used the revised Perdew-BurkeErnzerhof generalized gradient approximation (GGA) PBEsol exchange-correlation functional [53]. Interactions between core and valence electrons were described using the projector augmented wave (PAW) method [54], with cores of $[\mathrm{He}]$ for $\mathrm{Li},[\mathrm{Ne}]$ for $\mathrm{P},[\mathrm{Ne}]$ for $\mathrm{S},[\mathrm{Ne}]$ for $\mathrm{Cl}$, and $[\mathrm{Kr}]$ for I. Zero-pressure volumes were calculated for ordered $\mathrm{Li}_{6} \mathrm{PS}_{5} \mathrm{I}$ and $\mathrm{Li}_{6} \mathrm{PS}_{5} \mathrm{Cl}$, with the $4 c$ sites occupied by $\mathrm{S}$, and the $4 a$ sites occupied by $\mathrm{I}$ or $\mathrm{Cl}$. These calculations consisted of full geometry optimisations for a single unit cell (52 atoms) starting from the Materials Project structure ID-985592 [55], with a cut-off of $700 \mathrm{eV}$, and a $2 \times 2 \times 2$ Monkhorst-Pack $k$-point mesh. The optimised lattice parameters were then used to construct $2 \times 2 \times 2$ supercells (416 atoms) for the subsequent molecular dynamics simulations.

The molecular dynamics simulations used a plane-wave cut-off of $280 \mathrm{eV}$ and only the gamma point for $k$-space sampling. All MD simulations were performed at $500 \mathrm{~K}$, and used a time-step of $2 \mathrm{fs}$. For both $\mathrm{Li}_{6} \mathrm{PS}_{5} \mathrm{I}$ and $\mathrm{Li}_{6} \mathrm{PS}_{5} \mathrm{Cl}$ we have considered three different $\mathrm{S} / X$ configurations: $0 \%$ site-inversion, with $\mathrm{S}$ fully occupying the $4 c$ sites and $X$ fully occupying the $4 a$ sites, corresponding to the experimentally reported ordered $\mathrm{Li}_{6} \mathrm{PS}_{5} \mathrm{I}$ structure; $50 \%$ site-inversion, with a random $\mathrm{S} / X$ configuration that approximates the experimentally reported disordered $\mathrm{Li}_{6} \mathrm{PS}_{5} \mathrm{Cl}$ structure; and $100 \%$ site-inversion, with S fully occupying the $4 a$ sites and $X$ fully occupying the $4 c$ sites. The same randomly generated $50 \%$ siteinverted $\mathrm{S} / X$ configuration was used for the $\mathrm{Li}_{6} \mathrm{PS}_{5} \mathrm{I} 50 \%$ and $\mathrm{Li}_{6} \mathrm{PS}_{5} \mathrm{Cl} 50 \%$ simulations. For each system, the lattice parameters were kept fixed to the zero-pressure $0 \%$ optimised values. For each MD simulation two equilibration stages were performed, first using a 2 ps NVE run with temperature rescaling every 50 steps, followed by a 2 ps NVT run. For each simulation, the production runs were $70 \mathrm{ps}$.

The analysis of MD simulation trajectories is often complicated by "trivial" thermal motions of the mobile ions and of the host framework. Here, we are interested in non-trivial lithium displacements that contribute to net lithium diffusion, rather than short-timescale vibrational motion. To help resolve the lithium-diffusion processes in our simulations, we have extracted a series of "inherent" structures [56-58] from each simulation trajectory by per- forming conjugate-gradient geometry optimisations for configurations selected every 50 time-steps. Each inherent structure represents a local minimum on the corresponding $3 N$-dimensional potential energy surface, and the sequences of inherent structures from a given simulation describe the non-trivial motion of lithium ions as they move between these local minima.

A dataset containing inputs and outputs for all DFT calculations supporting this study is available under the CC-BY-4.0 licence from the University of Bath Research Data Archive [59]. All code used to analyse the simulation trajectories and to generate the corresponding figures is available as a series of Jupyter notebooks [60] under the MIT licence. Our analysis used the MATPLOTLIB [61], NUMPY [62, 63], PYMATGEN [64, 65], SCIPY [66], TQDM [67], VASPPY [68], SITE-ANALYSIS [69], POLYHEDRAL-ANALYSIS [70], KINISI [71], and CRYSTALTORTURE [72] Python packages.

\section{RESULTS}

Lithium Mean-Squared Displacements. The rate at which individual lithium ions diffuse through a solid electrolyte is described by the lithium self-diffusion coefficient, which can be calculated from molecular dynamics simulations as the slope of the lithium mean-squared displacement (MSD) versus time, in the long time limit [73]. Fig. 3 shows calculated lithium mean-squared displacements for $\mathrm{Li}_{6} \mathrm{PS}_{5} \mathrm{I}$ and $\mathrm{Li}_{6} \mathrm{PS}_{5} \mathrm{Cl}$ with $0 \%, 50 \%$, and $100 \% \mathrm{~S} / X$ site inversion. For both $X=\mathrm{I}$ and $X=\mathrm{Cl}$, for the anion-ordered systems $(0 \%$ and $100 \%$ site inversion) the MSD initially increases, before plateauing at longer times, giving an effective lithium diffusion coefficient of zero. These plateaus indicate that in the anion-ordered systems the lithium ions do not diffuse freely, but instead are confined to small disconnected regions of space. The MSDs of the anion-disordered systems (50\% site inversion) show qualitatively different behaviour: these MSDs continually increase at long times, corresponding to non-zero diffusion coefficients and long-ranged lithium diffusion. These results are consistent with data from previous molecular dynamics simulations $[22,30-32]$, and highlight two interesting points. First, the diffusion behaviour is qualitatively the same for 

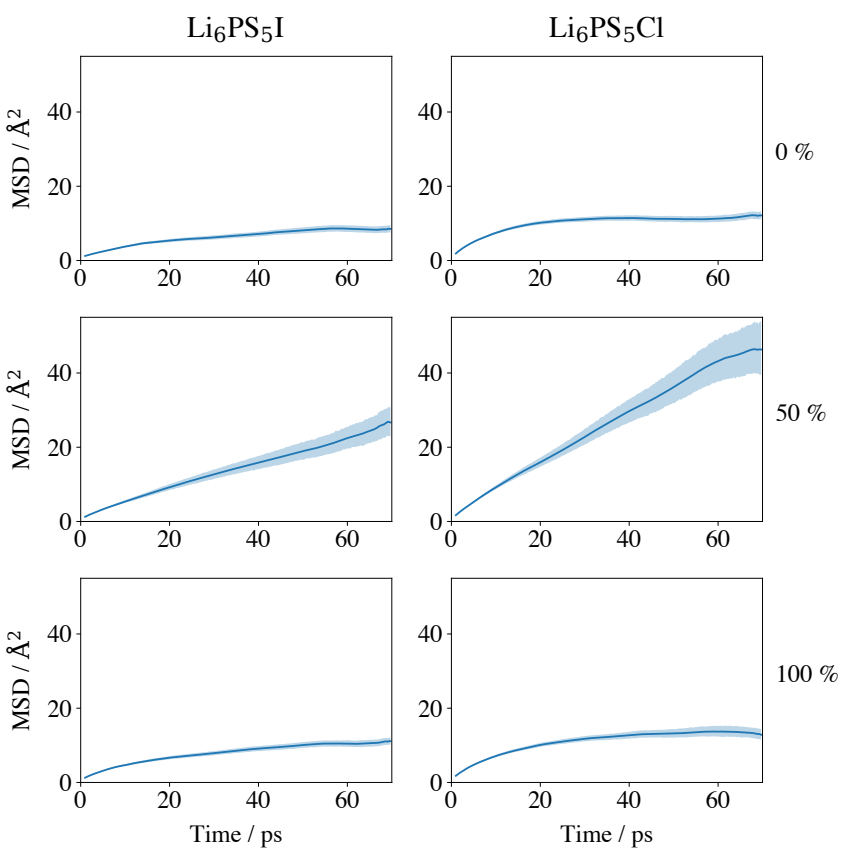

FIG. 3. Mean-squared displacement (MSD) of lithium ions for $\mathrm{Li}_{6} \mathrm{PS}_{5} \mathrm{I}$ and $\mathrm{Li}_{6} \mathrm{PS}_{5} \mathrm{Cl}$ with $0 \%, 50 \%$, and $100 \% \mathrm{~S} / X$ site inversion. Shaded regions show estimated $95 \%$ confidence intervals, calculated at each time interval via bootstrap sampling[74]. Source: The raw data and scripts used to generate this figure are available under CC-BY-4.0/MIT licences as part of Ref. [60].

$X=\mathrm{I}$ and $X=\mathrm{Cl}$, as noted previously by Stamminger et al [30]. Second, lithium caging is observed for both $0 \%$ and $100 \%$ site inversion, showing that long-ranged lithium diffusion is not a first-order consequence of occupying $4 a$ sites with sulfur, but that instead anion disorder across the $4 a$ and $4 c$ sites is the necessary prerequisite.

Tetrahedral Site Occupations. The qualitative difference in diffusion behaviour between anion-ordered and anion-disordered $\mathrm{Li}_{6} \mathrm{PS}_{5} X$ suggests that the arrangement of anions in each system directs the microscopic lithium diffusion mechanism. To examine the relationship between anion configuration and diffusion behaviour, we can calculate the time-averaged tetrahedral site-type populations for each simulation trajectory. To assign lithium ions to specific sites at each time-step, we use the instantaneous positions of the $\mathrm{S} / X$ anions to define the tetrahedra vertices. A lithium ion is deemed to occupy a particular tetrahedron if it sits inside the volume defined by these vertex positions [75].

Fig. 4 shows the time-averaged probabilities for a lithium ion to occupy each of the six tetrahedral site types, calculated using the inherent structures from each simulation trajectory. Each atomic configuration used in this analysis therefore corresponds to a local potential energy minimum. For all systems, lithium ions are most likely to occupy type 5 tetrahedra. This is broadly consistent with previous diffraction studies of $\mathrm{Li}_{6} \mathrm{PS}_{5} \mathrm{I}$
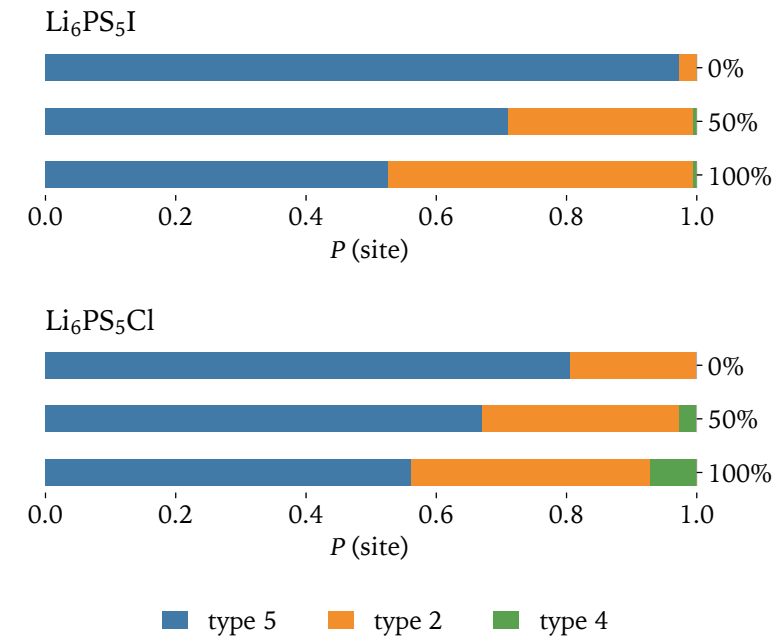

FIG. 4. Time-averaged probabilities for a lithium ion to occupy a particular tetrahedral site type, for (a) $\mathrm{Li}_{6} \mathrm{PS}_{5} \mathrm{I}$ and (b) $\mathrm{Li}_{6} \mathrm{PS}_{5} \mathrm{Cl}$ with $0 \%, 50 \%$, and $100 \% \mathrm{~S} / X$ site inversion. Source: The raw data and scripts used to generate this figure are available under CC-BY-4.0/MIT licences as part of Ref. [60].

(anion-ordered) and $\mathrm{Li}_{6} \mathrm{PS}_{5} \mathrm{Cl}$ (anion-disordered), which have assigned lithium as predominantly occupying two positions associated with the type 5 tetrahedra: the $48 \mathrm{~h}$ positions located inside each type 5 tetrahedron, and the $24 g$ positions (type 5 a sites) in the trigonal faces shared by type 5 tetrahedra pairs $[21,36,76]$.

In all six systems, we find some proportion of lithium ions located at non-type 5 tetrahedra. For $0 \%$ siteinverted $\mathrm{Li}_{6} \mathrm{PS}_{5} \mathrm{I}$, lithium partially occupies tetrahedra types 5 and 2 . In all the other systems lithium is distributed over tetrahedra types 5,2 , and 4 . The possibility of lithium occupying non-type 5 tetrahedra in $\mathrm{Li}_{6} \mathrm{PS}_{5} X$ argyrodites has been discussed in detail by Deiseroth et al. [36], who noted that lithium ions must pass through non-type 5 tetrahedra for long-ranged lithium transport to occur [45].

The observation of partial occupation of non-type 5 tetrahedra is qualitatively consistent with recent neutron diffraction studies of $\mathrm{Li}_{6} \mathrm{PS}_{5} \mathrm{Br}$ and $\mathrm{Li}_{6} \mathrm{PS}_{5} \mathrm{Cl}$, which have reported partial occupation of type 2 tetrahedra [46, 47]. The study of Minafra et al. also reported data for $\mathrm{Li}_{6} \mathrm{PS}_{5} \mathrm{I}$ [46], with lithium assigned only to type 5 and type $5 \mathrm{a}$ sites, in apparent contradiction to the simulation results presented here. Experimental samples of $\mathrm{Li}_{6} \mathrm{PS}_{5} \mathrm{I}$ are fully anion-ordered, and are approximated by our $0 \%$ site inversion model. For this system our simulations predict only $2.5 \%$ of Li occupies type 2 sites, which is unlikely to be resolved in diffraction experiments. Similarly, we predict very low numbers of lithium ions occupy type 4 sites, making direct experimental observation challenging.

In each system, the partially occupied tetrahedra define the set of pathways available for possible lithium diffusion. The relationship between the degree of anion- 


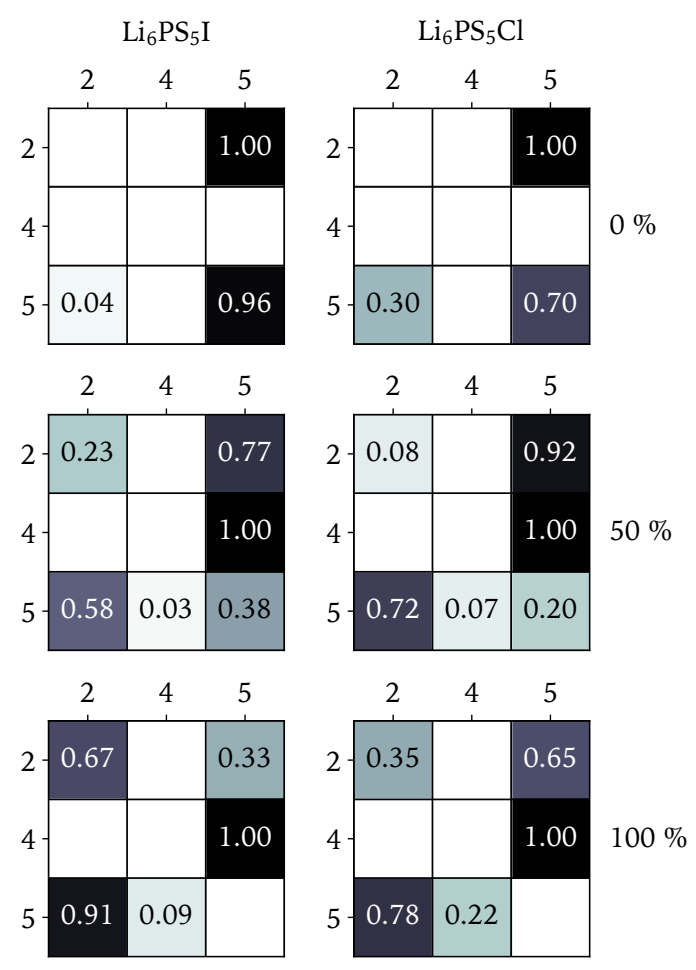

FIG. 5. Transition matrices showing the probabilities for lithium ions to move from site type $i$ (rows) to site type $j$ (columns), in 0\%,50\%, and $100 \%$ site-inverted $\mathrm{Li}_{6} \mathrm{PS}_{5} X$. Each row sums to a total probability of 1.0. Individual probabilities are shown rounded to 2 decimal places. Source: The raw data and scripts used to generate this figure are available under CC-BY-4.0/MIT licences as part of Ref. [60].

site inversion and lithium diffusion (cf. Fig. 3), however, is not explained by the varying occupations of these tetrahedral sites (Fig. 4). All six models predict partial occupation of both type 5 and type 2 tetrahedra, with type 2 occupation increasing with greater anion site-inversion. The set of all type 5 and type 2 tetrahedra forms a three-dimensional network of face-sharing tetrahedra, and we might therefore expect all systems to exhibit long-ranged lithium diffusion. Yet this is not the case, as only the $50 \%$ site-inverted systems exhibit long-ranged lithium diffusion. All systems apart from $0 \%$ site-inverted $\mathrm{Li}_{6} \mathrm{PS}_{5} \mathrm{I}$ also exhibit partial type 4 tetrahedral occupation, which further increases the connectivity of the three-dimensional tetrahedral network (Fig. 6(a)), and provides additional potential pathways for long-ranged diffusion. As is the case for increasing the occupation of type 2 tetrahedra, an increase in the occupation of the type 4 occupation is similarly not correlated with increased lithium diffusion. The $100 \%$ sitedisordered $\mathrm{Li}_{6} \mathrm{PS}_{5} X$ models have the highest probabilities of occupying both type 2 and type 4 tetrahedra, yet exhibit no long-ranged Li diffusion.

Site-Site Transition Probabilities. The lack of direct correlations between the tetrahedral site-type occupa- tions and the calculated diffusion data indicates that the varying capacities for long-ranged diffusion in the $\mathrm{Li}_{6} \mathrm{PS}_{5} X$ argyrodites is not a simple consequence of whether lithium does or does not partially occupy nontype 5 sites. Instead, we consider the possibility that it is not simply the occupations of the different tetrahedral sites that is important, but that the anion configuration may crucially affect whether the diffusion pathways connecting these sites are active or inactive. To determine the active diffusion paths in each system, we have analysed our inherent structure trajectories to identify transition events, defined as a lithium ion moving from one tetrahedral site to a neighbouring site. We can then calculate the probability that a lithium ion initially occupying site type $i$ subsequently moves to another site of type $j$, averaged over all observed transitions, for each $i \rightarrow j$ pairing. Fig. 5 shows transition matrices of the probabilities $P(i \rightarrow j)$. In each matrix, each row corresponds to a different initial site type $(2,4$, or 5$)$ and each nonblank entry in that row gives the observed probability of moving to a given adjacent site type. For $0 \%$ anion site-inversion, only $5 \rightarrow 5,5 \rightarrow 2$, and $2 \rightarrow 5$ transitions occur. With no $2 \rightarrow 2$ or $5 \rightarrow 4$ transitions, no long-ranged diffusion is possible, and lithium motion is restricted to closed "cages" around the $4 c$ sites (see Fig. 6(b)) [77]. For $100 \%$ site-inversion we observe only $2 \rightarrow 2,5 \rightarrow 2$, and $5 \rightarrow 4$ transitions. Long-ranged diffusion is now blocked by the inactive $5 \rightarrow 5$ transition, again giving to restricted lithium diffusion around the $4 a$ sites (see Fig. 6(c)). For $50 \%$ site-inversion, however, all jump types are observed, which is consistent with the existence of a contiguous diffusion network that can accommodate long-ranged $\mathrm{Li}$ diffusion; Li can now move around $4 a$ sites and $4 c$ sites. We therefore find that lithium motion between different tetrahedral sites is dependent on the local $\mathrm{S} / X$ anion configuration, which gives rise to a qualitative difference in active lithium diffusion pathways between anion-ordered and anion-disordered $\mathrm{Li}_{6} \mathrm{PS}_{5} X$ systems, as well as between models with $0 \%$ and $100 \%$ site inversion.

Time-Average Site Positions and Site-Site Percolation. As discussed in section II, all argyrodites possess topologically identical $\mathrm{MgCu}_{2}$-structured anions, and therefore have equivalent tetrahedral interstitial sites available for lithium diffusion. Understanding why a specific arrangement of anions across the $4 a$ and $4 c$ sites gives continuous versus discontinuous diffusion pathways requires going beyond the analysis presented above, which only considers the occupation of specific tetrahedra and the movement of lithium between these discrete sites. In the mixed-anion $\mathrm{Li}_{6} \mathrm{PS}_{5} X$ argyrodites, each tetrahedral hole may have a mixture of $\mathrm{S}$ and $X$ anions at its vertices, giving an asymmetric coordination environment. The equilibrium lithium position within a given tetrahedron therefore may not be located at the "ideal" tetrahedron centre. To understand how the $\mathrm{S} / X$ configuration affects the lithium substructure, we have calculated average lithium positions within each tetrahedron from our intrinsic structure trajectories. We have then considered 
(a)

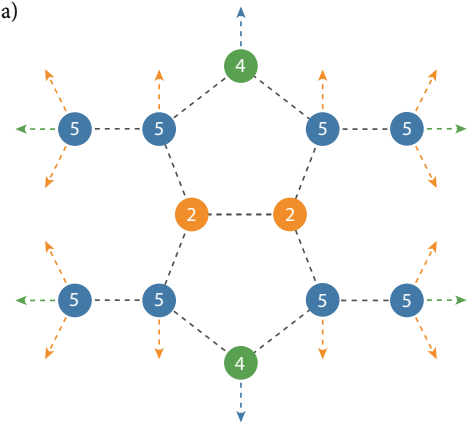

(b)

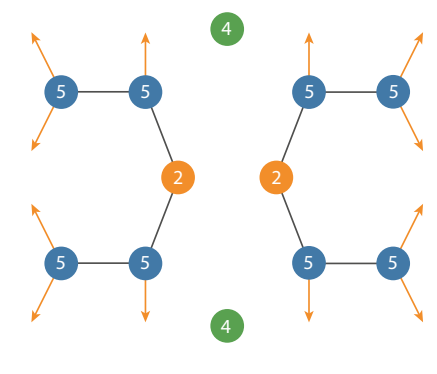

(c)

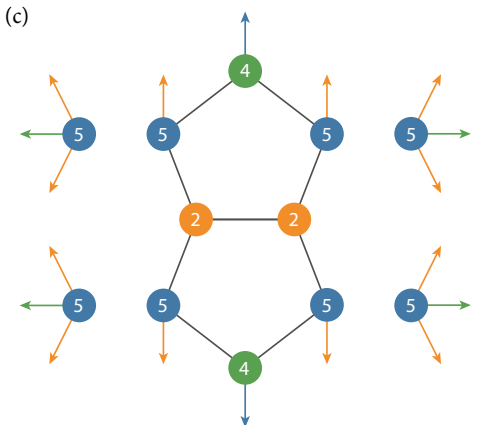

FIG. 6. (a) Schematic of all possible site-site lithium diffusion pathways between face-sharing pairs of type 2, type 4, and type 5 tetrahedra. (b) In $0 \%$ site-inverted $\mathrm{Li}_{6} \mathrm{PS}_{5} \mathrm{I}$ and $\mathrm{Li}_{6} \mathrm{PS}_{5} \mathrm{Cl}$, only $5 \rightarrow 5,5 \rightarrow 2$, and $2 \rightarrow 5$ transitions are observed, corresponding to "caged" diffusion around $4 c$ sites. (c) In $100 \%$ site-inverted $\operatorname{Li}_{6} \mathrm{PS}_{5} \mathrm{I}$ and $\mathrm{Li}_{6} \mathrm{PS}_{5} \mathrm{Cl}$, only $2 \rightarrow 2,2 \rightarrow 5,5 \rightarrow 2,5 \rightarrow 4$ and $4 \rightarrow 5$ transitions are observed, corresponding to "caged" diffusion around $4 a$ sites.

the distributions of site-site distances for each $\operatorname{Li}_{6} \mathrm{PS}_{5} X$ simulation. The radial distribution functions, $g(r)$, for specific pairs of these per-site average lithium positions, are shown in Fig. 7.

For the $0 \%$ site-inverted systems, the site-site rdfs show sharp peaks, indicating a degree of ordering by the lithium ions, and a clear hierarchy of site separations: the shortest $5-5$ separation is $\sim 1.2 \AA$, corresponding to pairs of adjacent type 5 tetrahedra. The next-nearest separation is $5-2$ at $\sim 1.5 \AA$, and the first $2-2$ site separation peak is at $>2 \AA$. The $100 \%$ site inverted systems also show sharp rdf peaks and distinct short and long sitesite separations. Now the nearest-neighbour distances increase in the order $2-2<4-5<2-5<5-5$, with the first $5-5$ peak at $>2 \AA$. Comparing the positions of nearestneighbour first peak for each site-pair to the corresponding site-site transition probabilities above (Fig. 5) shows long inter-site distances correspond to "inactive" diffusion paths, while short inter-site distances correspond to "active" diffusion paths. The site-site rdfs for the $50 \%$ site-inverted systems show broader distributions, indicating a range of site-site separations and a somewhat disordered lithium substructure. We find minimum inter-site distances of $<1 \AA$ for all four site-site distances. This does not mean that all site-site distances are this short in the $50 \%$ disordered system. Instead we observe a continuous range of short to long separations, indicating that these anion-disordered systems the average lithium positions within each site are statically disordered.

In each system, the hierarchy of site-site distances (Fig. 7) is correlated with the pattern of active and inactive lithium-site-transitions described in the previous section (Fig. 5). In the anion-ordered $0 \%$ and $100 \%$ site-inverted systems, tetrahedral pairs with short sitesite distances exhibit active transitions, while transitions between tetrahedral pair types with longer nearestneighbour distances are inactive. In the anion-disordered $50 \%$ site-inverted systems, all combinations of facesharing tetrahedral pairs exhibit a range of short and long site-site distances, and all site-site transitions are observed.

This correlation between short or long site-site distances and active or inactive lithium-site-transitions suggests a model wherein fast long-ranged lithium diffusion in the anion-disordered systems is associated with a percolating network of short lithium site separations, while non-diffusive motion in the anion-ordered systems is associated with a non-percolating disconnected network. To test this model, for each system we have calculated the minimum site - site separation distance at which the average lithium positions at each site form a percolating network (Fig. 7). We find this threshold percolation distance is significantly shorter for the anion-disordered systems than for the anion-ordered systems. For both the $0 \%$ and $100 \%$ site-inverted systems, the large $2-2$ or $5-5$ separations mean lithium motion is predominantly constrained to local "cages" of closely separated sites, surrounding the $4 c$ or $4 a$ positions respectively.

Anion-Lithium Radial Distribution Functions. The effect of ordered versus disordered anion configurations on the lithium substructure is also evident in the $\mathrm{S}(4 c / 4 a)$ $\mathrm{Li}$ and $X(4 a / 4 c)$-Li radial distribution functions (Fig. 8). In the anion-ordered $0 \%$ and $100 \%$ site-inverted systems, the nearest-neighbour S-Li distances are shorter than the nearest-neighbour $X-\mathrm{Li}$ distances. This corresponds to a displacement of the $\mathrm{Li}$ site positions towards the Soccupied $4 c$ or $4 a$ sites, and can be understood in terms of simple electrostatics - positive lithium ions are more strongly attracted to $\mathrm{S}^{2-}$ ions than to $X^{-}$ions. This asymmetry in anion-lithium coordination is larger for $X=\mathrm{I}$ than for $X=\mathrm{Cl}$, due to the additional difference in anion ionic radii. Because the $\mathrm{S}$ and $X$ anions are crystallographically ordered in these systems, a decrease of Li-site distances to S-occupied $4 c$ or $4 a$ sites corresponds to an increase of Li-site distances to $X$-occupied $4 a$ or $4 c$ sites, respectively. This pattern of short $\mathrm{S}-\mathrm{Li}$ and long $X$-Li distances, combined with the $\mathrm{S} / X$ ordering over $4 c$ and $4 a$ sites, explains the $\mathrm{Li}-\mathrm{Li}$ site distances discussed in the previous section (Fig. 7), which then explains the pattern of active and inactive lithium diffusion pathways 

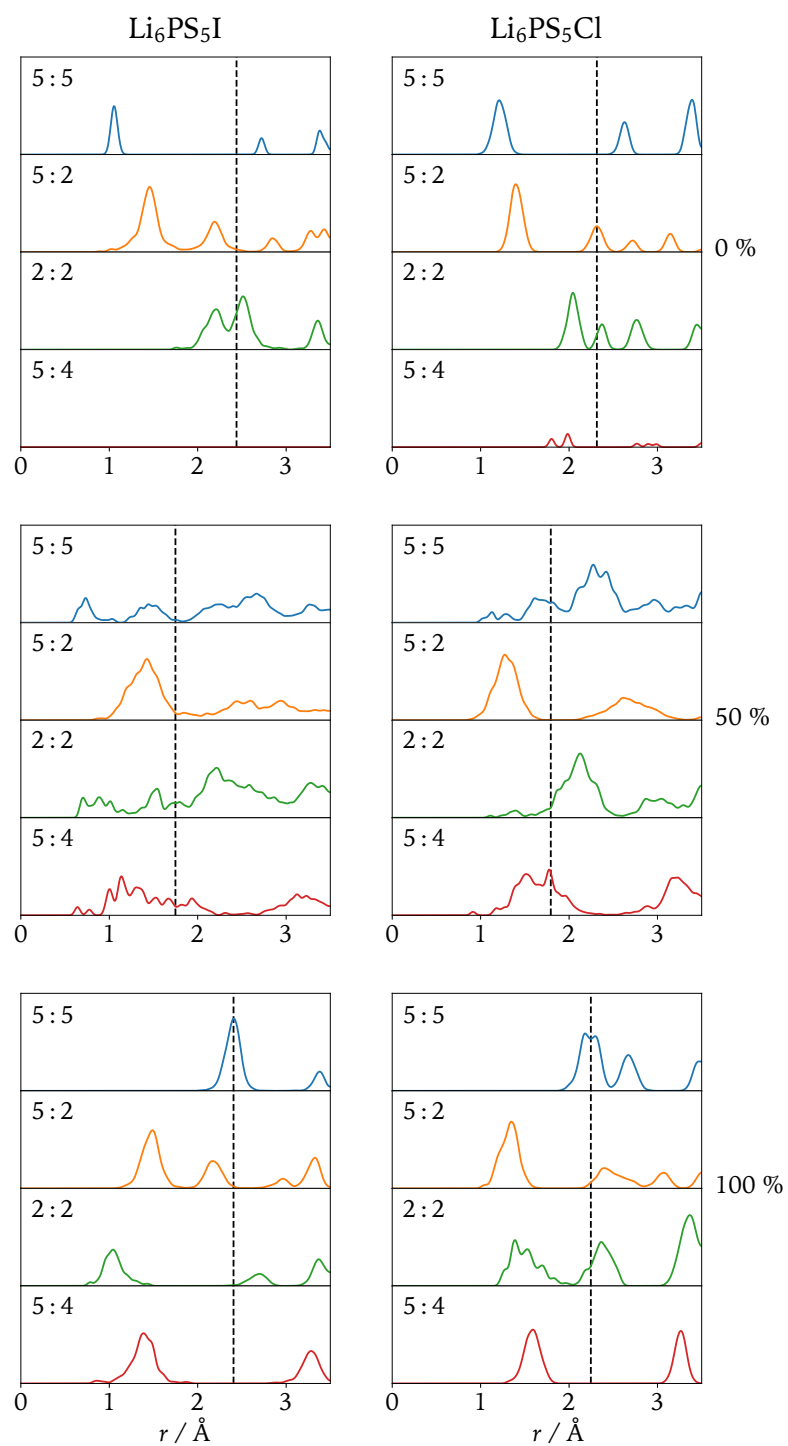

FIG. 7. Lithium site-site radial distribution functions for $\mathrm{Li}_{6} \mathrm{PS}_{5} \mathrm{I}$ and $\mathrm{Li}_{6} \mathrm{PS}_{5} \mathrm{Cl}$ at $0 \%, 50 \%$, and $100 \%$ anion-siteinversion. For each set of data, the vertical dashed line shows the minimum separation for which the lithium sites form a percolating network. Source: The raw data and scripts used to generate this figure are available under CC-BY-4.0/MIT licences as part of Ref. [60].

in these systems (Fig. 6). This effect, where an ordered $\mathrm{S} / X$ anion substructure induces ordered displacements in the lithium-site positions, is illustrated schematically in Fig. 10 ( $\mathrm{a}$ and b).

In the anion-disordered $50 \%$ site-inverted systems, the $\mathrm{S}-\mathrm{Li}$ and $X$-Li nearest-neighbour distances are more similar, and the corresponding peaks are broader. In particular, for $50 \%$ site-inverted $\mathrm{Li}_{6} \mathrm{PS}_{5} \mathrm{Cl}$ the $\mathrm{S}-\mathrm{Li}$ and $X-\mathrm{Li}$ nearest-neighbour peaks coincide. In an anion-disordered system, the Li sites experience a range of local coordination environments with different permutations of $\mathrm{S}$ and $X$ neighbouring anions. The average position of each
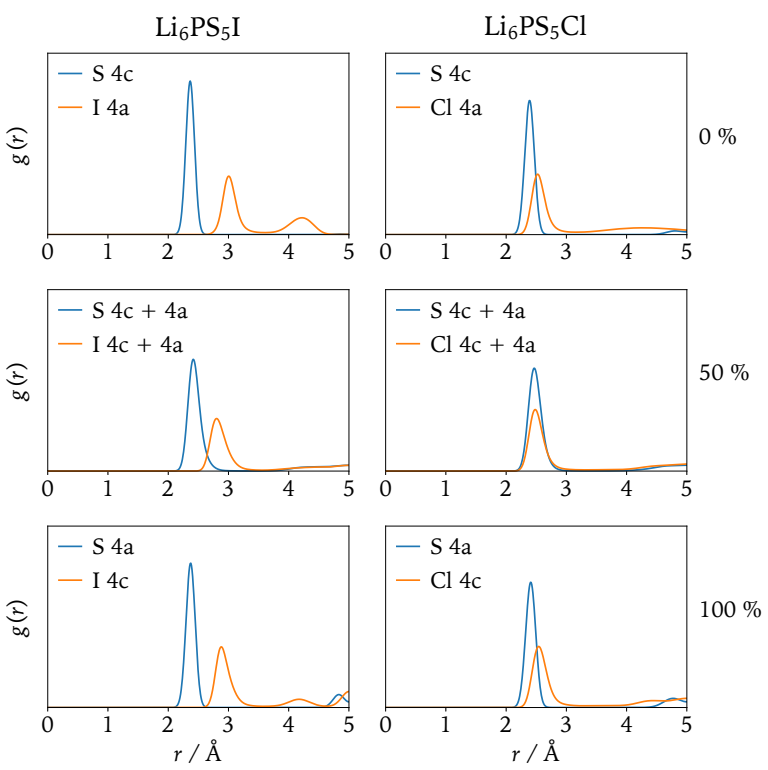

FIG. 8. $\mathrm{S}(4 a / 4 c)-\mathrm{Li}$ and $X(4 a / 4 c)-\mathrm{Li}$ radial distribution functions, $g(r)$, for $\mathrm{Li}_{6} \mathrm{PS}_{5} \mathrm{I}$ and $\mathrm{Li}_{6} \mathrm{PS}_{5} \mathrm{Cl}$ with $0 \%, 50 \%$, and $100 \% \mathrm{~S} / X$ site inversion. Source: The raw data and scripts used to generate this figure are available under CCBY-4.0/MIT licences as part of Ref. [60].

Li site now depends on the specific local anion environment. Because the $\mathrm{S}$ and $X$ anions are disordered, the arrangement of long Li site-site distances, which correspond to inactive diffusion paths, is also disordered, allowing a percolating network of shorter active diffusion paths (Fig. 10(c)).

The average numbers of lithium ions around the $\mathrm{S} / X$ $4 a$ and $4 c$ sites can be calculated by integrating the rdf data (Fig. 9). For $\mathrm{Li}_{6} \mathrm{PS}_{5} X$ stoichiometry argyrodites there are exactly six lithium ions per $4 a / 4 c \mathrm{~S}$ anion. For all the anion-ordered systems $(0 \%$ and $100 \%$ siteinversion) we find an average of $n=6$ lithium ions around the $4 c$ or $4 a \mathrm{~S}$ atoms, respectively, suggesting the structure can be described as $4 c$ or $4 a$-centered $\mathrm{SLi}_{6}$ sub-units, with $X$ occupying the remaining $4 a$ or $4 c$ sites. For the $50 \%$ systems, we find an average of $n>6 \mathrm{Li}$ ions associated with each $\mathrm{S}$ atom, suggesting a more complex lithium arrangement. Because the ratio of lithium to $4 a / 4 c$ sulfur is consistently $6 \mathrm{Li}$ to $1 \mathrm{~S}$ in all cases, an average coordination number of $n>6 \mathrm{Li}$ ions indicates that some $\mathrm{Li}$ contributes to coordination of more than one $\mathrm{S}$ centre.

Sulfur-Lithium Coordination Polyhedra. For a more detailed description of the local S-Li coordination environments, we have classified the local lithium coordination around each $4 a$ or $4 c$ sulfur according to the degree of geometric similarity with respect to a set of reference $\mathrm{SLi}_{x}$ coordination polyhedra. We consider a "coordination polyhedron" to consist of a single $\mathrm{S}$ atom residing at a $4 c$ or $4 a$ site plus the set of lithium ions within a spheri- 

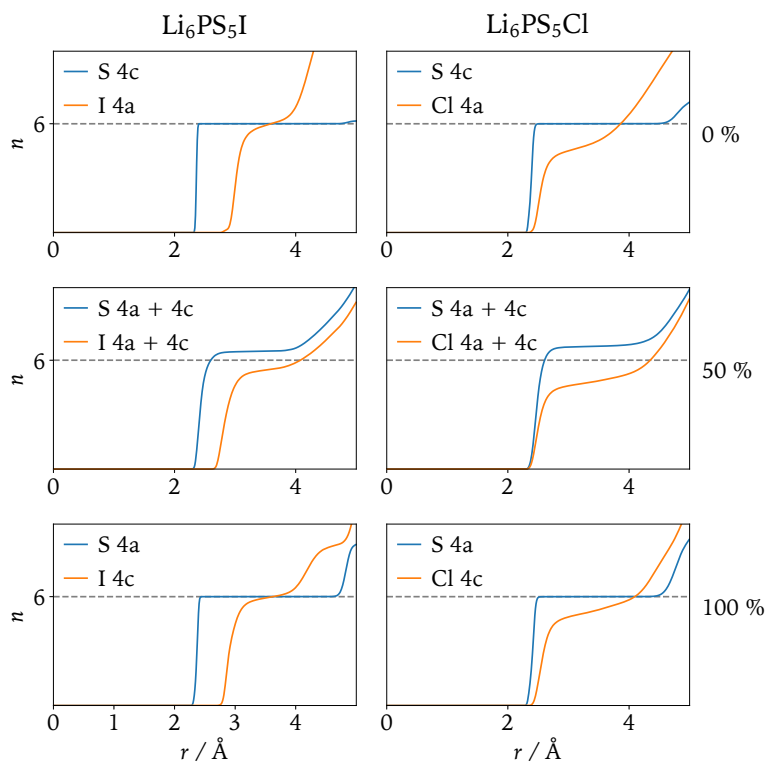

FIG. 9. $\mathrm{S}(4 a / 4 c)-\mathrm{Li}$ and $X(4 a / 4 c)-\mathrm{Li}$ coordination numbers, $n$, for $\mathrm{Li}_{6} \mathrm{PS}_{5} \mathrm{I}$ and $\mathrm{Li}_{6} \mathrm{PS}_{5} \mathrm{Cl}$ with $0 \%, 50 \%$, and $100 \% \mathrm{~S} / X$ site inversion. Source: The raw data and scripts used to generate this figure are available under CC-BY-4.0/MIT licences as part of Ref. [60].

cal cut-off $r_{\text {coord }}$, with the cut-off distance chosen to lie in the first plateau region from Fig. 9. For each coordination polyhedron, we can quantify the geometric similarity to a given reference polyhedron, such as a perfect octahedron, by calculating the corresponding Continuous Symmetry Measure (CSM) [78]. The CSM can be thought of as a normalised "distance" between two polyhedral geometries: larger CSM values indicate larger deviations from the reference geometry. Here, we classify each coordination polyhedron geometry by computing CSM values with respect to a set of common polyhedral coordination motifs $[65,79]$ and selecting the "most similar" motifgiven by the smallest CSM.

The relative proportions of different coordination polyhedra geometries are shown in Fig. 11. For the $0 \%$ and $100 \%$ site-inverted systems we observe nearly exclusively 6 -coordinate polyhedra [80], as suggested by the average S-Li coordination numbers (cf. Fig. 9). In these anionordered systems, these $\mathrm{SLi}_{6}$ units preferentially adopt approximately octahedral geometries, with a few trigonal prismatic configurations observed for all but the $\mathrm{Li}_{6} \mathrm{PS}_{5} \mathrm{I}$ $0 \%$ system. An octahedral distribution of lithium ions around each $\mathrm{S}$ is reasonable from electrostatics, because it minimises the net Coulomb repulsion between the lithium ions for $n=6$. For the anion-disordered systems we observe a mixture of 6 - and 7-coordinate polyhedra, in agreement with the average $n>6$ coordination number obtained from the $g(r)$ data.

$S-L i_{x}$ Polyhedra Dynamics. The $\mathrm{SLi}_{x}$ coordination polyhedra provide a schema for classifying the lithium dynamics in each system. For each polyhedron, we consider two features: the first is the set of lithium ions that define the polyhedron vertices, and the second is the set of edges that connect these vertices, which then defines the polyhedron topology. In our simulation trajectories, each lithium ion is assigned an integer index. The set of lithium ions that define a specific coordination polyhedron (all those within $r_{\text {coord }}$ of the central atom) can be described by a vertex list of these ion indices, e.g. (1, $3,7,20,52,100)$. The edge topology connecting these ions is described by an undirected edge graph, where we consider an edge formed between any two vertices of a polyhedron with a separation smaller than a threshold distance $r_{\text {edge }}$. These features allow us to define three classes of lithium motion:

1. Neither the vertex list nor the edge graph change, but the polyhedron undergoes a "rigid" rotation in space.

2. Only the edge graph changes. The vertex list remains unchanged. This corresponds to some internal reorganisation of lithium ions that changes the polyhedron topology.

3. The vertex list changes (and the edge graph therefore also changes). This corresponds to a lithium ion leaving a polyhedron (moving beyond the cutoff $r_{\text {coord }}$ ), or joining a new polyhedron, or both.

The first two of these correspond to local lithium motion, while the third constitutes lithium transfer between $\mathrm{SLi}_{x}$ polyhedra, which is required for long-ranged lithium diffusion.

In the anion-ordered $0 \%$ and $100 \%$ site-inverted systems, we find that exchange of lithium ions between coordination polyhedra is nearly never observed on the timescale of our simulation [81], which is consistent with the long-time plateaus in the lithium mean-squared displacement data and the inactive site-site transitions described above, and with data from previous molecular dynamics simulations of anion-ordered $\mathrm{Li}_{6} \mathrm{PS}_{5} X$ argyrodites $[22,30-33]$. In these anion-ordered systems, the lithium dynamics nearly exclusively comprises internal motions of $\mathrm{SLi}_{6}$ units.

For anion-ordered $\mathrm{Li}_{6} \mathrm{PS}_{5} \mathrm{I}$ (Fig. 12) these motions are predominantly rigid rotations of the $\mathrm{SLi}_{6}$ octahedral coordination polyhedra, which proceed via a concerted motion of four coplanar lithium ions around the perpendicular axis (Fig. 13(a)). We also observe a small number of internal reorganisations consisting of octahedral $\rightarrow$ trigonal-prismatic $\rightarrow$ octahedral transitions, which proceed via the concerted motion of three face-sharing lithium ions (Fig. 13(b)). This internal reorganisation via a trigonal-prismatic intermediate is analogous to a "Bailar twist" and is the minimum distortion pathway between two topologically inequivalent octahedra [82]. Both these motions have midpoints that are local potential energy minima, where the displaced lithium ions have moved from type 5 to type 2 tetrahedral sites. 
(a) "ideal" anion lattice

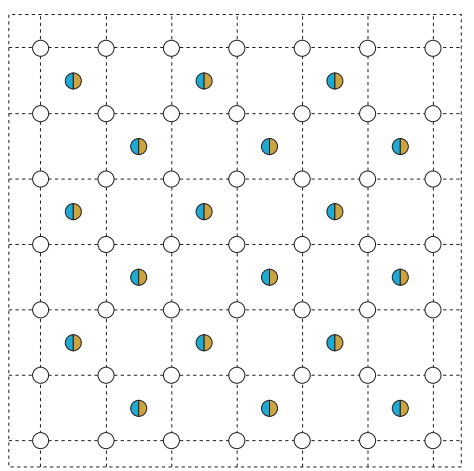

(b) ordered anion lattice

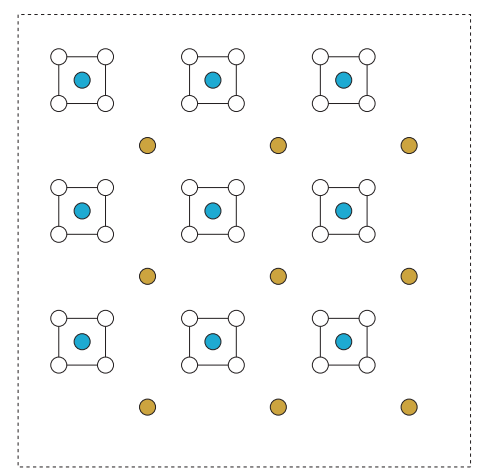

(c) disordered anion lattice

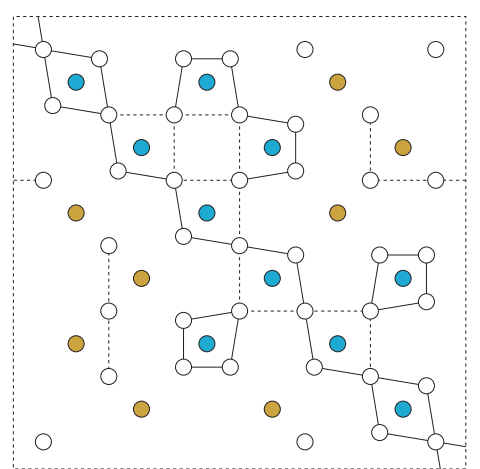

FIG. 10. Schematic illustrating the coupling between configurational order/disorder of the anion substructure and static order/disorder of the Li sites. (a) An "ideal" anion substructure. All Li sites are in symmetric environments, and all site-site distances are equal. (b) An ordered anion substructure. All Li sites are in locally asymmetric environments, and move towards $\mathrm{S}^{2-}$ (blue) and away from $X^{-}$(yellow) anions, to give an ordered non-percolating network of short site-site distances. (c) A disordered anion substructure. The shift of Li site positions depends on the local anion configuration. Moving towards $\mathrm{S}$ and away from $X$ anions produces a disordered percolating network of short site-site distances. Solid lines in (c) indicate site-site distances that are shorter than in the "ideal" anion substructure.
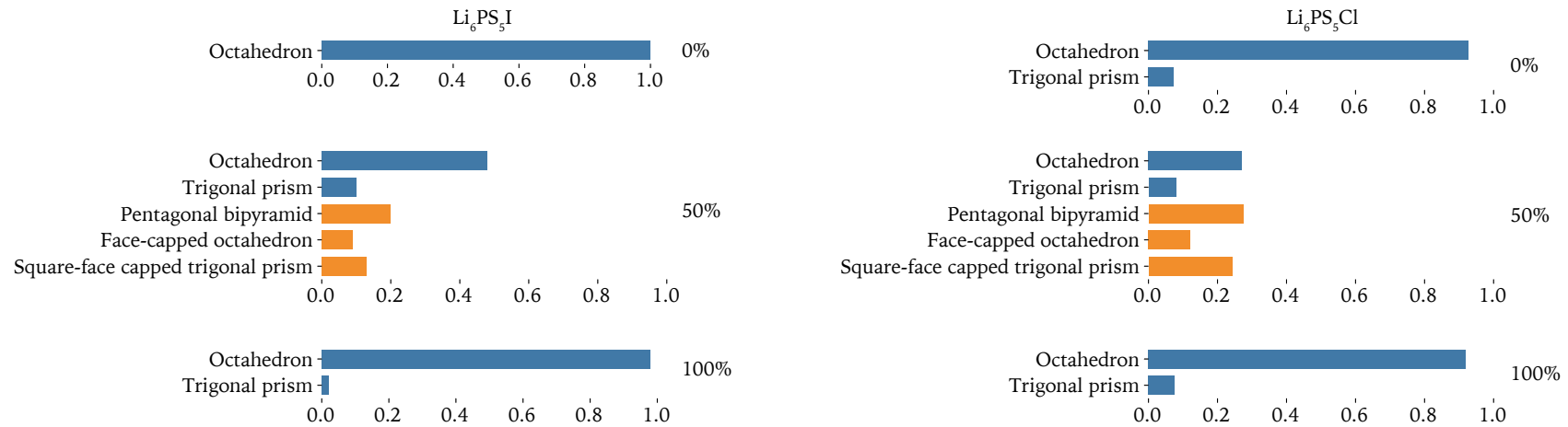

FIG. 11. Populations of $\mathrm{SLi}_{x}$ coordination polyhedra geometries, for $\mathrm{Li}_{6} \mathrm{PS}_{5} \mathrm{I}$ and $\mathrm{Li}_{6} \mathrm{PS}_{5} \mathrm{Cl}$ with $0 \%, 50 \%$, and $100 \% \mathrm{~S} / X$ site inversion. Polyhedral geometries have been assigned by calculating the minimum Continuous Symmetry Measure (CSM) [78] for a set of reference coordination geometries [65,79]. Blue bars denote 6-coordinate polyhedra; yellow bars denote 7 -coordinate polyhedra. Source: The raw data and scripts used to generate this figure are available under CC-BY-4.0/MIT licences as part of Ref. [60].

For anion-ordered $\mathrm{Li}_{6} \mathrm{PS}_{5} \mathrm{Cl}$ we observe similar behaviour, with local Li dynamics comprising both rigid octahedral rotations and internal reorganisation via trigonal-prismatic intermediates. These local Li dynamics in $\mathrm{Li}_{6} \mathrm{PS}_{5} \mathrm{Cl}$ are more frequent than in $\mathrm{Li}_{6} \mathrm{PS}_{5} \mathrm{I}$, and individual $\mathrm{SLi}_{x}$ units spend more time in intermediate configurations, where some lithium ions occupy type 2 sites, making it difficult to classify discrete dynamical events.

For the anion-disordered $\mathrm{Li}_{6} \mathrm{PS}_{5} \mathrm{I}$ and $\mathrm{Li}_{6} \mathrm{PS}_{5} \mathrm{Cl}$ systems we find qualitatively different behaviour to the anion-ordered systems described above. With $\mathrm{S} / X$ disorder present, lithium ions undergo rapid exchange between $\mathrm{SLi}_{x}$ units. This, again, is consistent with the meansquared displacements and site-site transition analyses presented above, and with previous molecular dynamics simulations [22, 30-33].
String-like Collective Diffusion. The conventional model for ionic diffusion in solid electrolytes assumes that ion transport is effected by a sequence of singleion "hops" between discrete sites [83-85], and this model has been assumed in the analysis of diffusion in lithium argyrodites in a number of previous studies [9, 11, 2225, 28, 41-44, 48, 86]. For many fast-ion solid electrolytes, however, ion transport instead proceeds via collective diffusion processes, whereby multiple ions participate in synchronous cooperative motion [87-97]. Such cooperative motions can be considered a defining characteristic of "superionic" conductivity, in distinction to fast, but conventional, single-particle-hopping [89], and concerted lithium diffusion has recently been proposed to be a contributing factor in the exceptionally high ionic conductivities of Li-excess $\mathrm{Li}_{6+x} M_{x} \mathrm{Sb}_{1-x} \mathrm{~S}_{5} \mathrm{I}(M=$ $\mathrm{Si}, \mathrm{Sn}, \mathrm{Ge}$ ) argyrodites [48]. 


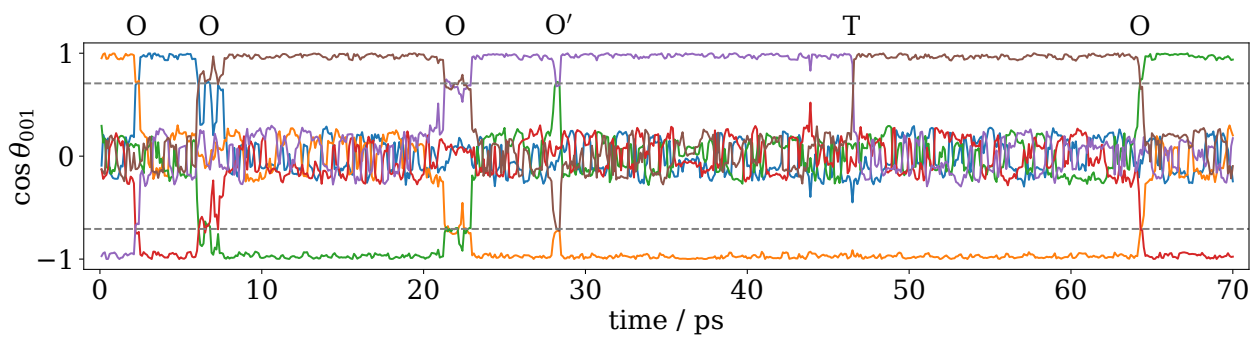

FIG. 12. Internal dynamics of one $4 c \mathrm{SLi}_{6}$ coordination polyhedron in the $\mathrm{Li}_{6} \mathrm{PS}_{5} \mathrm{I} 0 \%$ site-inverted system at $500 \mathrm{~K}$. The ordinate gives the normalised projection of each $\mathrm{S}-\mathrm{Li}$ vector onto the [001] cell axis (i.e. the cosine similarity). The horizontal dashed lines show $\cos \left( \pm 45^{\circ}\right)$. Labels above the plot indicate different classes of internal dynamics: O indicates a "rigid" octahedral rotation (Fig. 13(a)); $\mathrm{O}^{\prime}$ is an "incomplete" rotation, that moves to a $45^{\circ}$ rotated orientation, before returning to the previous geometry; $\mathrm{T}$ indicates an internal reorganisation via a trigonal-prismatic intermediate (Fig. 13(b)). Both the octahedral rotations and trigonal-prismatic rearrangements have stable intermediates where multiple lithium ions occupy type 2 tetrahedra. Source: The raw data and scripts used to generate this figure are available under CC-BY-4.0/MIT licences as part of Ref. [60].

(a) "rigid" octahedral rotation

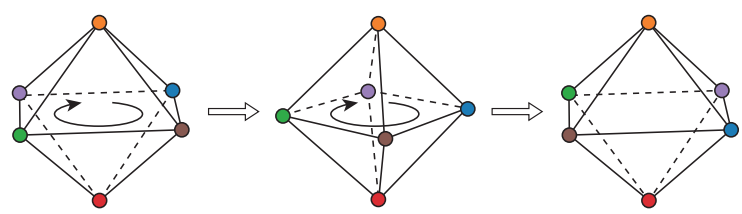

(b) trigonal-prismatic internal reorganisation

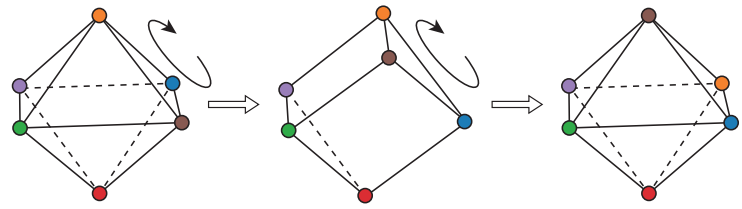

FIG. 13. Schematic of the concerted (a) rigid rotation and (b) trigonal-prismatic reorganisation modes of lithium motion within $\mathrm{SLi}_{6}$ coordination polyhedra in the anion-ordered $\mathrm{Li}_{6} \mathrm{PS}_{5} X$ systems.

Our site-site transition analysis, above, allowed a characterisation of the connectivity of active diffusion pathways within the argyrodite structure, and how the topology of the diffusion network varies with the $4 a / 4 c \mathrm{~S} / X$ anion configuration. That analysis, however, cannot distinguish between a single-particle hopping mechanism, and a collective "superionic" diffusion mechanism. In the anion-ordered system, we have already observed that the internal dynamics of $\mathrm{SLi}_{6}$ coordination polyhedra is highly concerted - octahedral rotations and trigonalprismatic reorganisations both involve synchronous motion of groups of lithium ions. For the anion-disordered systems, however, the more complex lithium dynamics means that this local "lithium-coordination" analysis is less useful in distinguishing between individual or concerted lithium motion.

To determine whether the lithium ions in aniondisordered $\mathrm{Li}_{6} \mathrm{PS}_{5} X$ systems diffuse via individual or col- lective processes, we have identified groups of lithium ions that are involved in cooperative string-like motions [98]. We define strings that form on a timescale $\Delta t$ by connecting two mobile ions $i$ and $j$ if

$$
\min \left[\left|\mathbf{r}_{i}(t+\Delta t)-\mathbf{r}_{j}(t)\right|,\left|\mathbf{r}_{j}(t+\Delta t)-\mathbf{r}_{i}(t)\right|\right]<\delta .
$$

This corresponds to selecting pairs of mobile ions where one ion has moved into a position previously occupied by the second ion. To construct strings we then connect ion pairs that occur within the same time window that contain one common mobile ion.

The identification of strings of mobile ions, via equation 1 , is not on its own sufficient to distinguish between individual hopping and a concerted diffusion mechanism. In the case of simple vacancy hopping, a sequence of "vacancy hops" produces a string of mobile ions, even though in this case the single-ion hops that produce this sequence are temporally uncorrelated. To distinguish a temporally uncorrelated process from a correlated process, we consider the distribution of string lengths observed in time $\Delta t$. For a stochastic hopping process, the number of hops in time window $\Delta t$, and hence the distribution of string lengths, samples a Poisson distribution [99]. Observing a probability distribution of string lengths that strongly deviates from a Poisson distribution is therefore evidence for ion displacements that are clustered in time.

Fig. 14 shows the probability distributions of string lengths, $P(n)$, from our simulations, for $\Delta t=5 \mathrm{ps}$. For the anion-ordered systems we find high probabilities of strings with lengths 2-4, corresponding to the concerted motions of ions within $\mathrm{SLi}_{6}$ coordination polyhedra described above. For the $50 \%$ site-inverted systems we observe a range of string lengths, with $P(n)$ following an approximate geometric distribution. This mirrors the behaviour observed in supercooled glassy liquids [98] where string-like diffusion is often associated with dynamic heterogeneity [56, 100-102], whereby spatially correlated subsets of particles exhibit much faster dynamics than 

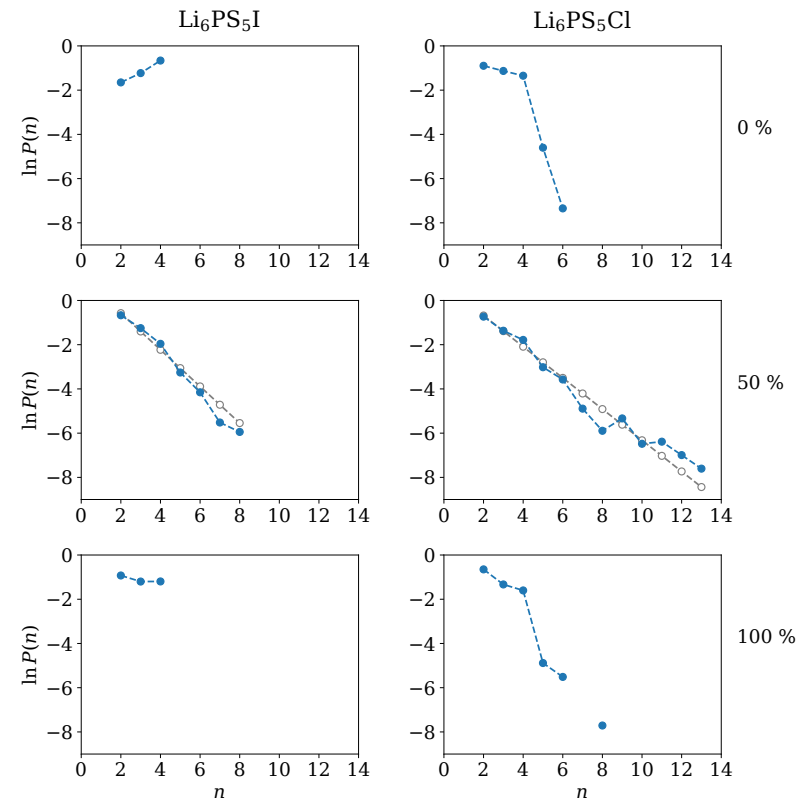

FIG. 14. Probability distributions $P(n)$ of string lengths $n$ for $\mathrm{Li}_{6} \mathrm{PS}_{5} \mathrm{I}$ and $\mathrm{Li}_{6} \mathrm{PS}_{5} \mathrm{Cl}$ with $0 \%, 50 \%$, and $100 \% \mathrm{~S} / X$ site inversion. For the $50 \%$ site-inverted systems, the grey empty symbols show the maximum likelihood estimate for a geometric distribution for each dataset. Source: The raw data and scripts used to generate this figure are available under CC-BY-4.0/MIT licences as part of Ref. [60].

the system average. A geometric distribution of string lengths is consistent with a mechanistic model consisting of string "initiation", followed by string "propagation" with the probability of a string increasing in length from $N$ particles to $N+1$ particles is independent of $N$. Our analysis here indicates that lithium mobility is effected by concerted ion motions in all our systems. In the anionordered systems, these motions are "closed-loops" typically consisting of groups of four or three lithium ions undergoing local cyclic motions (cf. Fig. 13). In the aniondisordered systems, we find string-like concerted motions that facilitate the diffusion of extended groups of lithium ions.

\section{SUMMARY AND DISCUSSION}

The data from our molecular dynamics simulations provide multiple complementary perspectives of the atomic-scale diffusion behaviour in $\mathrm{Li}_{6} \mathrm{PS}_{5} X$ argyrodites. Combining these perspectives provides the basis of a coherent model for the lithium diffusion mechanism in these systems, and how this is affected with the degree of substitutional $4 a / 4 c$ anion disorder.

We first analysed the lithium distribution in each simulation in terms of occupation of the different tetrahedral sites within the close-packed anion substructure. We find that lithium ions predominantly occupy type
5 tetrahedral sites; particularly so for the fully ordered $\mathrm{S}(4 c) / X(4 a)$ systems; which broadly agrees with previous diffraction experiments $[21,36]$. For all systems, we also find partial occupation of non-type 5 tetrahedra. Dieseroth et al. have previously noted that long-ranged diffusion of lithium through the argyrodite structure requires movement of lithium through non type- 5 tetrahedra [45]. In more recent studies of lithium argyrodites, however, the lithium substructure has typically been considered purely in terms of occupation of type 5 (and 5a) sites, with lithium diffusion decomposed into classes of direct $5 \rightarrow 5$ transitions $[9,11,22,24,25,27,30,32,33,41$, $42,86,103,104]$. This simplified perspective neglects the different tetrahedra types defined by the close-packed anion substructure, and obscures their roles in the lithium diffusion processes in different argyrodite compositions. For $\mathrm{Li}_{6} \mathrm{PS}_{5} X$ systems, these non-type 5 tetrahedra are not simply high-energy intermediates that define local potential energy maxima along lithium diffusion $5 \rightarrow 5$ pathways. Instead we find stable Li configurations, corresponding to local potential-energy minima, in which non-type 5 sites are occupied. This illustrates the importance of these sites when describing the relevant potential energy surface for lithium diffusion. Occupation of non-type 5 tetrahedra has been identified in experimental samples for $x(\mathrm{Li})>6$ argyrodites [48-50] as well as in recent neutron diffraction studies of $\mathrm{Li}_{6} \mathrm{PS}_{5} \mathrm{Br}$ and $\mathrm{Li}_{6} \mathrm{PS}_{5} \mathrm{Cl}[46,47]$. The tetrahedrally close-packed geometry defined by the $\mathrm{MgCu}_{2}$-structured anion sites is common to all argyrodites, and we therefore expect analyses that consider diffusion in terms of ion motion between these different tetrahedra to provide useful insight into the transport mechanisms operating in argyrodite stoichiometries beyond $\mathrm{Li}_{6} \mathrm{PS}_{5} X$.

In the case of the $\mathrm{Li}_{6} \mathrm{PS}_{5} X$ systems, an analysis in terms of occupation of spatially-discrete lithium sites also allows the active lithium diffusion pathways in each system to be resolved. While the partial site-occupations for these anion-ordered and anion-disordered systems might equally suggest the existence of a contiguous lithium-diffusion pathway, via a network of face-sharing tetrahedra, lithium does not always move freely between these partially occupied tetrahedra. For anionordered systems, there are regular "blocked" pathways between specific neighbouring tetrahedral sites, giving a non-contiguous diffusion network. Our analysis of the time-average lithium positions within each partially occupied tetrahedral site shows that "blocked" pathways correspond to large average $\mathrm{Li}-\mathrm{Li}$ separations, while the set of short "active" pathways forms closed orbits around the $4 c$ or $4 a \mathrm{~S}$ anions, producing the restricted "cage-like" lithium diffusion reported in previous studies $[22,30,31,34]$. This can be considered a form of lithium ordering, induced by the configurational anion order, that arises from a preference for shorter S-Li than $X-\mathrm{Li}$ distances. In the anion-disordered systems, conversely, we find no systematic pattern of short-long average lithium separations between adjacent sites: the set 
of short "active" pathways is disordered and forms a percolating three-dimensional network, that facilitates longranged lithium diffusion.

The idea that the lithium ions are, in some sense, ordered when the $\mathrm{S} / X$ anions are substitutionally ordered, but that lithium ions are disordered when these anions are disordered, and that this difference directs the lithium diffusion behaviour, also emerges from our analysis of the local lithium coordination environment around the $4 a$ and $4 c \mathrm{~S}$ anions. In the anion-ordered systems the lithium substructure can be considered as 6 -coordinate $\mathrm{SLi}_{x}$ polyhedra around the $4 c$ or $4 a$ sulfur atoms. This "pseudo-ordering" corresponds to an effective crystal symmetry where the regular 6-coordinate $\mathrm{SLi}_{x}$ motif is invariant under integer lattice-vector translations, even though the lithium ions are crystallographically disordered over the available tetrahedral sites.

The pseudo-ordering of lithium in $\mathrm{S} / X$-ordered argyrodites can be explained by considering the various Coulombic interactions between $\mathrm{S}-\mathrm{Li}, X-\mathrm{Li}$, and $\mathrm{Li}-\mathrm{Li}$ ion pairs. Lithium ions are attracted more strongly to $\mathrm{S}^{2-}$ than $X^{-}$anions, due to the larger formal charge of S, which encourages $\mathrm{Li}$ to adopt cage-like configurations around the $4 a$ or $4 c \mathrm{~S}$ ions. The repulsive $\mathrm{Li}-\mathrm{Li}$ Coloumbic interactions, however, tend to maximise $\mathrm{Li}-\mathrm{Li}$ separations within each coordination cage. In an anionordered system, these two factors can be simultaneously optimised by arranging exactly six Li ions around each $4 a$ or $4 c \mathrm{~S}$ anion (Fig. 15(a)). Within each $\mathrm{SLi}_{6}$ unit, the $\mathrm{Li}-\mathrm{Li}$ repulsion is minimised by the $\mathrm{Li}$ ions adopting an approximately octahedral configuration. Lithium motions that only produce internal reorganisation of individual $\mathrm{SLi}_{6}$ units do not disrupt this pseudo-ordering, and are frequent on a simulation timescale. These motions are highly cooperative, proceeding via octahedral or trigonal-prismatic intermediates that preserve the mutual $\mathrm{Li}-\mathrm{Li}$ separation.

Long-ranged diffusion, in contrast, requires lithium motion between adjacent $\mathrm{SLi}_{x}$ coordination polyhedra. Consider two adjacent $\left[\mathrm{SLi}_{6}+\mathrm{SLi}_{6}\right]$ polyhedra in the ordered system. Lithium transfer between these polyhedra produces a $\left[\mathrm{SLi}_{5}+\mathrm{SLi}_{7}\right]$ configuration, and disrupts the preferred pseudo-ordered coordination motif. This "disordered" configuration is energetically disfavoured, because of the increased $\mathrm{Li}-\mathrm{Li}$ repulsion within the $\mathrm{SLi}_{7}$ unit. Forming $\mathrm{SLi}_{x}$ coordination environments with $x \neq 6$ in these anion-ordered $\mathrm{Li}_{6} \mathrm{PS}_{5} X$ argyrodites can therefore be considered a form of defect-pair formation, analogous to Frenkel pair formation in stoichiometric crystals [99].

In the $\mathrm{S} / X$-disordered systems, we can again understand the behaviour by considering the interplay of anion- $\mathrm{Li}$ and $\mathrm{Li}-\mathrm{Li}$ Coulomb interactions. $\mathrm{Li}$ ions again preferentially coordinate $\mathrm{S}$, rather than $X$ anions. We have seen in the anion-ordered system that if these $\mathrm{SLi}_{x}$ units are well-separated, the $\mathrm{Li}-\mathrm{Li}$ interactions between $\mathrm{SLi}_{x}$ units are negligible, and the total electrostatic energy is minimised by forming a set of regular octahe- dral $\mathrm{SLi}_{6}$ subunits. In an anion-disordered system, however, $\mathrm{S}$ ions occupy both $4 a$ and $4 c$ sites. Forming adjacent $\mathrm{SLi}_{6}$ octahedra would now introduce short Li$\mathrm{Li}$ distances between $\mathrm{Li}$ ions nominally associated with the different $\mathrm{S}$ anions (Fig. 15(b)): $\mathrm{SLi}_{6}$ configurations can be said to be "geometrically frustrated" [105]. To avoid these short $\mathrm{Li}-\mathrm{Li}$ separations, $\mathrm{Li}$ ions can instead be "shared" between adjacent $\mathrm{SLi}_{x}$ environments, producing a mixture of $\mathrm{SLi}_{6}$ and $\mathrm{SLi}_{7}$ coordination motifs, and spatially disordered average Li positions within each tetrahedral site (Fig. 15(c)). Lithium motion between different S-coordination environments is now possible without changing the net distribution of coordination environments - e.g. Li transfer between two adjacent $\mathrm{SLi}_{x}$ environments might proceed as $\mathrm{SLi}_{7}+\mathrm{SLi}_{6} \rightarrow \mathrm{SLi}_{6}$ $+\mathrm{SLi}_{7}$. This is expected to correspond to a low energy diffusion process, analogous to diffusion of extrinsic defects (vacancies or interstitials) in a non-stoichiometric conventional crystal [99].

Superionic conductivity in solid electrolytes is often associated with some form of disorder within the mobileion substructure [106-109], and studies of various solid electrolyte families have revealed a range of mechanisms that can contribute to this substructural disorder $[10,32,105,110-114]$. The results presented here provide another example of this general principle. In this instance, superionic conductivity arises as a consequence of substitutional disorder within the immobile ion host-substructure, via induced mobile-ion disorder. Enhanced conductivities have been reported in other materials with configurational host-framework disorder [115119], and the same underlying mechanism of induced mobile-ion disorder may be responsible in these cases. This raises the question of the extent to which substitutional framework-disorder might be useful as a general design strategy to obtain superionic conductivity in other families of solid electrolytes?

In the case of the lithium argyrodites, we can ask the more specific question of how these results for $\mathrm{Li}_{6} \mathrm{PS}_{5} X$ might suggest design strategies for optimising the ionic conductivity of $x(\mathrm{Li}) \neq 6$ argyrodites? Our results indicate that in the case of $\mathrm{Li}_{6} \mathrm{PS}_{5} X$, substitutional anion disorder, and the resulting lithium disorder, are key to achieving fast Li diffusion. This suggests that substitutional disorder; either due to mixed anions, or to partial substitution of $\mathrm{P}$ with elements such as $\mathrm{Ge}$, might have a similar positive effect in $x(\mathrm{Li}) \neq 6$ systems. A disordered potential energy surface may promote fast diffusion by causing concerted "superionic" diffusion mechanisms [120], rather than slower independent-hopping diffusion mechanisms. A second, contrasting, interpretation comes from noting that in $\mathrm{Li}_{6} \mathrm{PS}_{5} X$ systems, $\mathrm{S} / X$ anion disorder gives $\mathrm{S}$ occupying adjacent $4 a$ and $4 c$ sites, which contributes to lithium disorder due to Coulombic frustration of otherwise regular $\mathrm{SLi}_{x}$ units. This perspective suggests that in $x(\mathrm{Li}) \neq 6$ argyrodites, fast lithium-ion diffusion might be achieved in compositions in which the $\mathrm{S} / X$ ratio differs from one, making, for example $\mathrm{Li}_{7} \mathrm{PS}_{6}$ 
(a) $\mathrm{S} / \mathrm{X}$ ordered, with $\mathrm{SLi}_{6}$ polyhedra

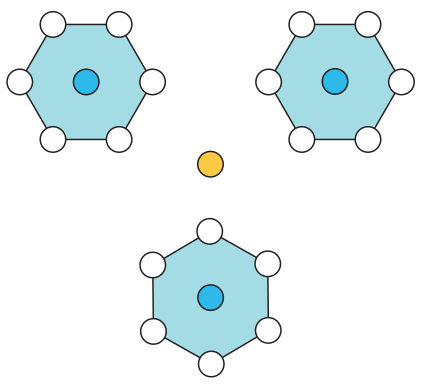

(b) $\mathrm{S} / \mathrm{X}$ disordered, with $\mathrm{SLi}_{6}$ polyhedra

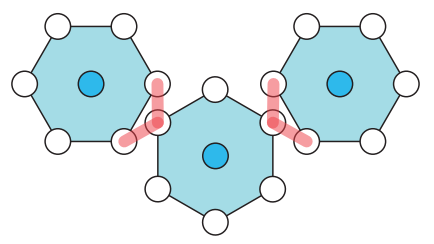

(c) $\mathrm{S} / \mathrm{X}$ disordered, with $\mathrm{SLi}_{\{6,7\}}$ polyhedra

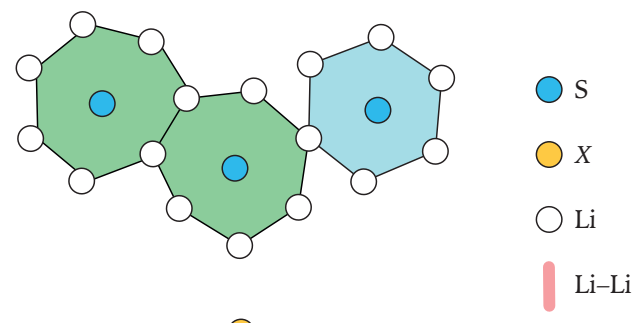

FIG. 15. Schematic of the change in lithium coordination around $4 a / 4 c \mathrm{~S}$ ions as a function of $\mathrm{S} / X$ order $/$ disorder. (a) For $\mathrm{S} / X$ anion ordering, the $4 c$ (or $4 a$ ) $\mathrm{S}$ ions are well separated, and Li ions arrange into $\mathrm{SLi}_{x}$ coordination "cages". (b) $\mathrm{S} / X$ anion disorder means S ions occupy adjacent $4 a$ and $4 c$ sites. Maintaining SLi 6 coordination would produce short Li-Li distances (red arrows), which are Coulombically disfavoured. (c) Sharing Li between adjacent $4 a$ and $4 c$ S coordination environments reduces the net $\mathrm{Li}-\mathrm{Li}$ repulsion, and gives a mix of irregular $\mathrm{SLi}_{6}$ and $\mathrm{SLi}_{7}$ coordination environments.

an interesting end-member case.

Finally, we note that the highest room-temperature ionic conductivities for lithium argyrodites have been reported for "Li excess" systems with $x(\mathrm{Li})>6$, such as $\mathrm{Li}_{6.6} \mathrm{P}_{0.4} \mathrm{Ge}_{0.6} \mathrm{~S}_{5} \mathrm{I}$ [11] and $\mathrm{Li}_{6+x} M_{x} \mathrm{Sb}_{1-x} \mathrm{~S}_{5} \mathrm{I} \quad(M=$ $\{\mathrm{Si}, \mathrm{Sn}, \mathrm{Ge}\})[48]$. In the latter case, lithium has been shown to occupy non-type 5 sites, which was attributed to the $x(\mathrm{Li})>6$ lithium stoichiometry, with this lithium "site-disorder" suggested as the origin of the observed fast lithium-ion conduction [48]. This suggestion is consistent with the general principle that lithium disorder (in some form) is necessary to achieve fast lithium diffusion, and raises the possibility of doing so directly through control of lithium stoichiometry; in contrast to the induced lithium disorder arising from substitutional framework disorder, as for the anion-disordered $\mathrm{Li}_{6} \mathrm{PS}_{5} X$ systems. Attributing the exceptional ionic conductivity of these experimental $x(\mathrm{Li})>6$ systems to a single mechanistic origin is challenging. The excess Li stoichiometry is a consequence of aliovalent substitution of hostframework atoms occupying the $4 b$ site, which introduces a new source of substitutional disorder within the host framework. These materials also exhibit small amounts of $\mathrm{S} / I$ disorder, which may also contribute to Li disorder and enhance Li diffusion [11]. Resolving the interplay between stoichiometry, structure, and lithium dynamics, and using this understanding to further optimise the ionic conductivities of this family of solid electrolytes, presents an intriguing challenge. The complexity of these issues suggests that a full understanding will only be reached by combining data from systematic experimental studies of controlled stoichiometries with insight from correspond- ing computational studies.

\section{ACKNOWLEDGEMENTS}

B. J. M. acknowledges support from the Royal Society (UF130329 \& URF $\backslash \mathrm{R} \backslash 191006$ ). Additional support was received from the Faraday Institution (grant no. FIRG003). Calculations were performed using the Balena High Performance Computing Service at the University of Bath, and using the ARCHER supercomputer, with access through membership of the UK's HPC Materials Chemistry Consortium, funded by EPSRC Grant No. EP/L000202. B. J. M. thanks Wolfgang Zeier for useful discussions.

\section{SUPPORTING INFORMATION}

Comparison of unprocessed simulation trajectories versus inherent structure trajectories for sites analysis.

\section{DATA AVAILABILITY}

A dataset containing inputs and outputs for all DFT calculations supporting this study is available under the CC-BY-4.0 licence from the University of Bath Research Data Archive [59]. All code used to analyse the simulation trajectories and to generate the corresponding figures is available as a series of Jupyter notebooks as Ref. [60] under the MIT licence.
[1] Z. Zhang, Y. Shao, B. Lotsch, Y.-S. Hu, H. Li, J. Janek, L. F. Nazar, C.-W. Nan, J. Maier, M. Armand, and L. Chen, New horizons for inorganic solid state ion con- ductors, Energy Environ. Sci. 11, 1945 (2018).

[2] J. C. Bachman, S. Muy, A. Grimaud, H.-H. Chang, N. Pour, S. F. Lux, O. Paschos, F. Maglia, S. Lupart, 
P. Lamp, L. Giordano, and Y. Shao-Horn, Inorganic solid-state electrolytes for lithium batteries: Mechanisms and properties governing ion conduction, Chem. Rev. 116, 140 (2016).

[3] S. Ohno, A. Banik, G. F. Dewald, M. A. Kraft, T. Krauskopf, N. Minafra, P. Till, M. Weiss, and W. G. Zeier, Materials design of ionic conductors for solid state batteries, Prog. Energy 2, 022001 (2020).

[4] T. Famprikis, P. Canepa, J. A. Dawson, M. S. Islam, and C. Masquelier, Fundamentals of inorganic solidstate electrolytes for batteries, Nat. Mater. 21, 1 (2019).

[5] J. Janek and W. G. Zeier, A solid future for battery development, Nature Energy 1, 1 (2016).

[6] S. P. Culver, R. Koerver, T. Krauskopf, and W. G. Zeier, Designing ionic conductors: The interplay between structural phenomena and interfaces in thiophosphatebased solid-state batteries, Chem. Mater. 30, 4179 (2018).

[7] A. G. Squires, D. O. Scanlon, and B. J. Morgan, Native defects and their doping response in the lithium solid electrolyte $\mathrm{Li}_{7} \mathrm{La}_{3} \mathrm{Zr}_{2} \mathrm{O}_{12}$, Chem. Mater. 32, 1876 (2019).

[8] T. Fuchs, S. P. Culver, P. Till, and W. G. Zeier, Defect-mediated conductivity enhancements in $\mathrm{Na}_{3-x} \mathrm{Pn}_{1-x} \mathrm{~W}_{x} \mathrm{~S}_{4}(\mathrm{Pn}=\mathrm{P}, \mathrm{Sb})$ using aliovalent substitutions, ACS Energy Lett. 5, 146 (2020).

[9] R. Schlem, M. Ghidiu, S. P. Culver, A.-L. Hansen, and W. G. Zeier, Changing the static and dynamic lattice effects for the improvement of the ionic transport properties within the argyrodite $\mathrm{Li}_{6} \mathrm{PS}_{5-x} \mathrm{Se}_{x} \mathrm{I}$, ACS Appl. Energy Mater. 3, 9 (2019).

[10] L. Zhou, A. Assoud, A. Shyamsunder, A. Huq, Q. Zhang, P. Hartmann, J. Kulisch, and L. F. Nazar, An entropically stabilized fast-ion conductor: $\mathrm{Li}_{3.25}\left[\mathrm{Si}_{0.25} \mathrm{P}_{0.75}\right] \mathrm{S}_{4}$, Chem. Mater. 31, 7801 (2019).

[11] M. A. Kraft, S. Ohno, T. Zinkevich, R. Koerver, S. P. Culver, T. Fuchs, A. Senyshyn, S. Indris, B. J. Morgan, and W. G. Zeier, Inducing high ionic conductivity in the lithium superionic argyrodites $\mathrm{Li}_{6+x} \mathrm{P}_{1-x} \mathrm{Ge}_{x} \mathrm{~S}_{5} \mathrm{I}$ for all-solid-state batteries, J. Am. Chem. Soc. 140, 16330 (2018).

[12] Y. Zhang, X. He, Z. Chen, Q. Bai, A. M. Nolan, C. A. Roberts, D. Banerjee, T. Matsunaga, Y. Mo, and C. Ling, Unsupervised discovery of solid-state lithium ion conductors, Nature Comm. 10, 1 (2019).

[13] L. Kahle, A. Marcolongo, and N. Marzari, Highthroughput computational screening for solid-state Liion conductors, Energy Environ. Sci. 13, 928 (2020).

[14] S. Muy, J. Voss, R. Schlem, R. Koerver, S. J. Sedlmaier, F. Maglia, P. Lamp, W. G. Zeier, and Y. Shao-Horn, High-throughput screening of solid-state Li-ion conductors using lattice-dynamics descriptors, ISCIENCE 16, 270 (2019).

[15] A. D. Sendek, E. D. Cubuk, E. R. Antoniuk, G. Cheon, Y. Cui, and E. J. Reed, Machine learning-assisted discovery of solid Li-ion conducting materials, Chem. Mater. 31, 342 (2018).

[16] T. Krauskopf, S. P. Culver, and W. G. Zeier, Bottleneck of diffusion and inductive effects in $\mathrm{Li}_{10} \mathrm{Ge}_{1-x} \mathrm{Sn}_{x} \mathrm{P}_{2} \mathrm{~S}_{12}$, Chem. Mater. 30, 1791 (2018).

[17] M. Weiss, D. A. Weber, A. Senyshyn, J. Janek, and W. G. Zeier, Correlating transport and structural properties in $\mathrm{Li}_{1+x} \mathrm{Al}_{x} \mathrm{Ge}_{2-x}\left(\mathrm{PO}_{4}\right)_{3}$ LAGP prepared from aqueous solution, ACS Appl. Mater. Int. 10, 10935
(2018).

[18] T. Krauskopf, S. Muy, S. P. Culver, S. Ohno, O. Delaire, Y. Shao-Horn, and W. G. Zeier, Comparing the descriptors for investigating the influence of lattice dynamics on ionic transport using the superionic conductor $\mathrm{Na}_{3} \mathrm{PS}_{4-x} \mathrm{Se}_{x}$, J. Am. Chem. Soc. 140, 14464 (2018).

[19] M. Burbano, D. Carlier, F. Boucher, B. J. Morgan, and M. Salanne, Sparse cyclic excitations explain the low ionic conductivity of stoichiometric $\mathrm{Li}_{7} \mathrm{La}_{3} \mathrm{Zr}_{2} \mathrm{O}_{12}$, Phys. Rev. Lett. 116, 135901 (2016).

[20] S. P. Culver, A. G. Squires, N. Minafra, C. W. F. Armstrong, T. Krauskopf, F. Böcher, C. Li, B. J. Morgan, and W. G. Zeier, Evidence for a solidelectrolyte inductive effect in the superionic conductor $\mathrm{Li}_{10} \mathrm{Ge}_{1-x} \mathrm{Sn}_{x} \mathrm{P}_{2} \mathrm{~S}_{12}$, J. Am. Chem. Soc. 142, 21210 (2020).

[21] H.-J. Deiseroth, S.-T. Kong, H. Eckert, J. Vannahme, C. Reiner, T. Zaiß, and M. Schlosser, $\mathrm{Li}_{6} \mathrm{PS}_{5} X:$ A class of crystalline Li-rich solids with an unusually high $\mathrm{Li}^{+}$ mobility, Angew. Chem. Int. Edit. 47, 755 (2008).

[22] N. J. J. de Klerk, I. Rosłoń, and M. Wagemaker, Diffusion mechanism of $\mathrm{Li}$ argyrodite solid electrolytes for Liion batteries and prediction of optimized halogen doping: The effect of Li vacancies, halogens, and halogen disorder, Chem. Mater. 28, 7955 (2016).

[23] M. A. Kraft, S. P. Culver, M. Calderon, F. Böcher, T. Krauskopf, A. Senyshyn, C. Dietrich, A. Zevalkink, J. Janek, and W. G. Zeier, Influence of lattice polarizability on the ionic conductivity in the lithium superionic argyrodites $\mathrm{Li}_{6} \mathrm{PS}_{5} X(X=\mathrm{Cl}, \mathrm{Br}, \mathrm{I}), \mathrm{J}$. Am. Chem. Soc. 139, 10909 (2017).

[24] N. Minafra, S. P. Culver, T. Krauskopf, A. Senyshyn, and W. G. Zeier, Effect of Si substitution on the structural and transport properties of superionic Liargyrodites, J. Mater. Chem. A 6, 645 (2018).

[25] P. Adeli, J. D. Bazak, K. H. Park, I. Kochetkov, A. Huq, G. R. Goward, and L. F. Nazar, Boosting solid-state diffusivity and conductivity in lithium superionic argyrodites by halide substitution, Angew. Chem. Int. Edit. 58, 8681 (2019).

[26] R. P. Rao and S. Adams, Studies of lithium argyrodite solid electrolytes for all-solid-state batteries, Phys. Stat. Sol. A 208, 1804 (2011).

[27] P. R. Rayavarapu, N. Sharma, V. K. Peterson, and $\mathrm{S}$. Adams, Variation in structure and $\mathrm{Li}^{+}$-ion migration in argyrodite-type $\mathrm{Li}_{6} \mathrm{PS}_{5} X(X=\mathrm{Cl}, \mathrm{Br}, \mathrm{I})$ solid electrolytes, J. Solid State Electrochem. 16, 1807 (2012).

[28] I. Hanghofer, M. Brinek, S. L. Eisbacher, B. Bitschnau, M. Volck, V. Hennige, I. Hanzu, D. Rettenwander, and H. M. R. Wilkening, Substitutional disorder: structure and ion dynamics of the argyrodites $\mathrm{Li}_{6} \mathrm{PS}_{5} \mathrm{Cl}$, $\mathrm{Li}_{6} \mathrm{PS}_{5} \mathrm{Br}$ and $\mathrm{Li}_{6} \mathrm{PS}_{5} \mathrm{I}$, Phys. Chem. Chem. Phys. 21, 8489 (2019).

[29] H. M. Chen, C. Maohua, and S. Adams, Stability and ionic mobility in argyrodite-related lithium-ion solid electrolytes, Phys. Chem. Chem. Phys. 17, 16494 (2015).

[30] A. R. Stamminger, B. Ziebarth, M. Mrovec, T. Hammerschmidt, and R. Drautz, Ionic conductivity and its dependence on structural disorder in halogenated argyrodites $\mathrm{Li}_{6} \mathrm{PS}_{5} X(X=\mathrm{Br}, \mathrm{Cl}, \mathrm{I})$, Chem. Mater. 31, 8673 (2019).

[31] Z. Deng, Z. Zhu, I.-H. Chu, and S. P. Ong, Datadriven first-principles methods for the study and design 
of alkali superionic conductors, Chem. Mater. 29, 281 (2016).

[32] A. Gautam, M. Sadowskic, N. Prinzd, H. Eickhoffe, N. Minafra, M. Ghidiu, S. P. Culver, K. Albe, T. F. Fässler, M. Zobel, and W. G. Zeier, Rapid crystallization and kinetic freezing of site-disorder in the lithium superionic argyrodite $\mathrm{Li}_{6} \mathrm{PS}_{5} \mathrm{Br}$, Chem. Mater. 31, 10178 (2019).

[33] A. Baktash, J. C. Reid, T. Roman, and D. J. Searles, Diffusion of lithium ions in lithium-argyrodite solid-state electrolytes, npj Comput. Mater. 6, 10.1038/s41524-020-00432-1 (2020).

[34] X. Feng, P.-H. Chien, Y. Wang, S. Patel, P. Wang, H. Liu, M. Immediato-Scutto, and Y.-Y. Hu, Enhanced ion conduction by enforcing structural disorder in Lideficient argyrodites $\mathrm{Li}_{6-x} \mathrm{PS}_{5-x} \mathrm{Cl}_{1+x}$, Energy Stor. Mater. 30, 67 (2020).

[35] J. von Unterrichter and K.-J. Range, $\mathrm{Ag}_{8} \mathrm{GeTe}_{6}$, ein vertreter der argyroditfamilie, Z. Naturforsch. 33 b, 866 (1978).

[36] S.-T. Kong, H.-J. Deiseroth, C. Reiner, Ö. Gün, E. Neumann, C. Ritter, and D. Zahn, Lithium argyrodites with phosphorus and arsenic: Order and disorder of lithium atoms, crystal chemistry, and phase transitions, Chem. Eur. J. 16, 2198 (2010).

[37] W. F. Kuhs, R. Nitsche, and K. Scheunemann, The argyrodites - a new family of tetrahedrally close-packed structures, Mat. Res. Bull 14, 241 (1979).

[38] F. C. Frank and J. S. Kasper, Complex alloy structures regarded as sphere packings. I. definitions and basic principles, Acta. Cryst. 11, 184 (1958).

[39] F. C. Frank and J. S. Kasper, Complex alloy structures regarded as sphere packings. II analysis and classification of representative structures, Acta. Cryst. 12, 483 (1959).

[40] C. Bonneau and M. O'Keefe, Intermetallic crystal structures as foams. Beyond Frank-Kasper, Inorg. Chem. 54, 808 (2014).

[41] T. Bernges, S. P. Culver, N. Minafra, R. Koerver, and W. G. Zeier, Competing structural influences in the Li superionic conducting argyrodites $\mathrm{Li}_{6} \mathrm{PS}_{5-x} \mathrm{Se}_{x} \mathrm{Br}(0 \leq$ $x \leq 1)$ upon Se substitution, Inorg. Chem. 57, 13920 (2018).

[42] S. Ganapathy, C. Yu, E. R. H. van Eck, and M. Wagemaker, Peeking across grain boundaries in a solid-state ionic conductor, ACS Energy Lett. 4, 1092 (2019).

[43] I. Hanghofer, B. Gadermaier, and H. M. R. Wilkening, Fast rotational dynamics in argyrodite-type $\mathrm{Li}_{6} \mathrm{PS}_{5} X$ $(X: \quad \mathrm{Cl}, \mathrm{Br}, \mathrm{I})$ as seen by ${ }^{31} \mathrm{P}$ nuclear magnetic relaxation - on cation-anion coupled transport in thiophosphates, Chem. Mater. 31, 1 (2019).

[44] P. Wang, H. Liu, S. Patel, X. Feng, P.-H. Chien, Y. Wang, and Y.-Y. Hu, Fast ion conduction and its origin in $\mathrm{Li}_{6-x} \mathrm{PS}_{5-x} \mathrm{Br}_{1+x}$, Chem. Mater. 32, 3833 (2020).

[45] H.-J. Deiseroth, J. Maier, K. Weichert, V. Nickel, S.T. Kong, and C. Reiner, $\mathrm{Li}_{7} \mathrm{PS}_{6}$ and $\mathrm{Li}_{6} \mathrm{PS}_{5} X(X: \mathrm{Cl}$, $\mathrm{Br}, \mathrm{I})$ : Possible three-dimensional diffusion pathways for lithium ions and temperature dependence of the ionic conductivity by impedance measurements, Z. Anorg. Allg. Chem. 637, 1287 (2011).

[46] N. Minafra, M. A. Kraft, T. Bernges, C. Li, R. Schlem, B. J. Morgan, and W. G. Zeier, Local charge inhomogeneity and lithium distribution in the superionic ar- gyrodites $\operatorname{Li}_{6} \mathrm{PS}_{5} x(X=\mathrm{Cl}, \mathrm{Br}, \mathrm{I})$, Inorg. Chem. 59, 11009 (2020).

[47] R. Schlenker, A.-L. Hansen, A. Senyshyn, T. Zinkevich, M. Knapp, T. Hupfer, H. Ehrenberg, and S. Indris, Structure and diffusion pathways in $\mathrm{Li}_{6} \mathrm{PS}_{5} \mathrm{Cl}$ argyrodite from neutron diffraction, pair-distribution function analysis and NMR, Chem. Mater. (2020).

[48] L. Zhou, A. Assoud, Q. Zhang, X. Wu, and L. F. Nazar, A new family of argyrodite thioantimonate lithium superionic conductors, J. Am. Chem. Soc. 141, 19002 (2019).

[49] W. Huang, K. Yoshino, S. Hori, K. Suzuki, M. Yonemura, M. Hirayama, and R. Kanno, Superionic lithium conductor with a cubic argyrodite-type structure in the Li-Al-Si-S system, Journal of Solid State Chemistry 270, 487 (2019).

[50] W. Huang, C. LinDong, S. Hori, K. Suzuki, M. Yonemura, M. Hirayama, and R. Kanno, Ionic conduction mechanism of a lithium superionic argyrodite in the LiAl-Si-S-O system, Mater. Adv. (2020).

[51] G. Kresse and J. Furthmüller, Efficient iterative schemes for $A b$ Initio total-energy calculations using a planewave basis set, Phys. Rev. B 54, 11169 (1996).

[52] G. Kresse and J. Furthmuller, Efficiency of ab-initio total energy calculations for metals and semiconductors using a plane-wave basis set, Comp. Mater. Sci. 6, 15 (1996).

[53] J. Perdew, A. Ruzsinszky, G. Csonka, O. Vydrov, G. Scuseria, L. Constantin, X. Zhou, and K. Burke, Restoring the density-gradient expansion for exchange in solids and surfaces, Phys. Rev. Lett. 100, 136406 (2008)

[54] G. Kresse and D. Joubert, From ultrasoft pseudopotentials to the projector augmented-wave method, Phys. Rev. B 59, 1758 (1999).

[55] K. Persson, Materials data on $\mathrm{Li}_{6} \mathrm{PS}_{5} \mathrm{Cl}$ (SG:216) by Materials Project (2016).

[56] A. S. Keys, L. O. Hedges, J. P. Garrahan, S. C. Glotzer, and D. Chandler, Excitations are localized and relaxation is hierarchical in glass-forming liquids, Phys. Rev. X 1, 021013 (2011).

[57] F. H. Stillinger and T. A. Weber, Packing structures and transitions in liquids and solids, Science 225, 983 (1984).

[58] A. Heuer, Exploring the potential energy landscape of glass-forming systems: From inherent structures via metabasins to macroscopic transport, J. Phys-Condens. Mat. 20 (2008).

[59] B. J. Morgan, DFT dataset for mechanistic origin of superionic lithium diffusion in anion-disordered $\mathrm{Li}_{6} \mathrm{PS}_{5} X$ argyrodites (2020), https://researchdata.bath.ac. uk/id/eprint/814.

[60] B. J. Morgan, Data analysis for "mechanistic origin of superionic lithium diffusion in anion-disordered $\mathrm{Li}_{6} \mathrm{PS}_{5} X$ argyrodites" (2020), https://github.com/ bjmorgan/data_argyrodite_disorder.

[61] J. D. Hunter, Matplotlib: A 2d graphics environment, Comput. Sci. Eng. 9, 90 (2007).

[62] T. E. Oliphant, A guide to NumPy (USA: Trelgol Publishing, 2006).

[63] S. van der Walt, S. C. Colbert, and G. Varoquaux, The NumPy array: A structure for efficient numerical computation, Computing in Science \& Engineering 13, 22 (2011). 
[64] S. P. Ong, W. D. Richards, A. Jain, G. Hautier, M. Kocher, S. Cholia, D. Gunter, V. L. Chevrier, K. A. Persson, and G. Ceder, Python materials genomics (pymatgen): A robust, open-source python library for materials analysis, Comp Mater. Sci 68, 314 (2013).

[65] D. Waroquiers, J. George, M. Horton, S. Schenk, K. A. Persson, G.-M. Rignanese, X. Gonze, and G. Hautier, ChemEnv: a fast and robust coordination environment identification tool, Acta. Cryst. B 76, 683 (2020).

[66] P. Virtanen, , R. Gommers, T. E. Oliphant, M. Haberland, T. Reddy, D. Cournapeau, E. Burovski, P. Peterson, W. Weckesser, J. Bright, S. J. van der Walt, M. Brett, J. Wilson, K. J. Millman, N. Mayorov, A. R. J. Nelson, E. Jones, R. Kern, E. Larson, C. J. Carey, I. Polat, Y. Feng, E. W. Moore, J. VanderPlas, D. Laxalde, J. Perktold, R. Cimrman, I. Henriksen, E. A. Quintero, C. R. Harris, A. M. Archibald, A. H. Ribeiro, F. Pedregosa, and P. van Mulbregt, SciPy 1.0: fundamental algorithms for scientific computing in python, Nature Methods 17, 261 (2020).

[67] C. O. da Costa-Luis, tqdm: A fast, extensible progress meter for Python and CLI, J. Open Source Soft. 4, 1277 (2019).

[68] B. J. Morgan, vasppy: A Python suite for manipulating VASP input and output, https://doi.org/10.5281/zenodo.1263753, https://github.com/bjmorgan/vasppy.

[69] site-analysis codebase: https://github.com/ bjmorgan/site-analysis.

[70] polyhedral-analysis codebase: https://github. com/bjmorgan/polyhedral-analysis.

[71] kinisi codebase: https://github.com/bjmorgan/ kinisi.

[72] C. O'Rourke and B. J. Morgan, crystal-torture: A crystal tortuosity module, J. Open Source Soft. 4, 1306 (2019).

[73] B. J. Morgan and P. A. Madden, Absence of a space-charge-derived enhancement of ionic conductivity in $\beta-\gamma$-heterostructured 7H- and 9R-AgI, J. PhysCondens. Matter 24, 275303 (2012).

[74] B. Efron, Bootstrap methods: Another look at the jackknife, Ann. Stat. 7, 1 (1979).

[75] Because the anion substructure is tetrahedrally closepacked, using anion-position-defined tetrahedra to define discrete sites guarantees every lithium ion is assigned to one, and only one, tetrahedral site at each simulation time-step.

[76] C. Yu, L. van Eijck, S. Ganapathy, and M. Wagemaker, Synthesis, structure and electrochemical performance of the argyrodite $\mathrm{Li}_{6} \mathrm{PS}_{5} \mathrm{Cl}$ solid electrolyte for Li-ion solid state batteries, Electrochimica Acta 215, 93 (2016).

[77] We note that the $2 \rightarrow 2$ and $5 \rightarrow 4 \rightarrow 5$ pathways correspond to the two "intercage" diffusion pathways proposed by Schlenker et al. on the basis of differential bond-valence analysis [47].

[78] M. Pinsky and D. Avnir, Continuous symmetry measures. 5. The classical polyhedra, Inorganic Chemistry 37, 5575 (1998).

[79] D. Waroquiers, X. Gonze, G.-M. Rignanese, C. WelkerNieuwoudt, F. Rosowski, M. Göbel, S. Schenk, P. Degelmann, R. André, R. Glaum, and G. Hautier, Statistical analysis of coordination environments in oxides, Chem. Mater. 29, 8346 (2017).
[80] For $\mathrm{Li}_{6} \mathrm{PS}_{5} \mathrm{I}$ with $0 \%$ and $100 \%$ site-inversion, $100 \%$ and $100 \%$ of the S-Li coordination polyhedra are 6coordinate, respectively. For $\mathrm{Li}_{6} \mathrm{PS}_{5} \mathrm{Cl}$ with $0 \%$ and $100 \%$ site-inversion, $99.98 \%$ and $99.57 \%$ of S-Li coordination polyhedra are 6 -coordinate, respectively.

[81] For the $0 \%$ and $100 \%$ site-inverted $\mathrm{Li}_{6} \mathrm{PS}_{5} \mathrm{I}$ systems, we observe no exchange of lithium between coordination polyhedra during the $70 \mathrm{ps}$ simulations. For $\mathrm{Li}_{6} \mathrm{PS}_{5} \mathrm{Cl}$ with $0 \%$ and $100 \%$ site-inversion, we observe Liexchange between coordination polyhedra with frequencies of 0.002 / polyhedron/ps and 0.019 /polyhedron/ps, respectively.

[82] D. Casanova, J. Cirera, M. Llunell, P. Alemany, D. Avnir, and S. Alvarez, Minimal distortion pathways in polyhedral rearrangements, J. Am. Chem. Soc. 126, 1755 (2004).

[83] B. J. Morgan, Lattice-geometry effects in garnet solid electrolytes: a lattice-gas Monte Carlo simulation study, R. Soc. Open Sci. 4, 170824 (2017).

[84] A. Van der Ven, J. Bhattacharya, and A. A. Belak, Understanding Li diffusion in Li-intercalation compounds, Acc. Chem. Res. 46, 1216 (2013).

[85] C. R. A. Catlow, Static lattice simulation of structure and transport in superionic conductors, Sol. Stat. Ionics 8, 89 (1983).

[86] S. Ohno, B. Helm, T. Fuchs, G. Dewald, M. A. Kraft, S. P. Culver, A. Senyshen, and W. G. Zeier, Further evidence for energy landscape flattening in the superionic argyrodites $\mathrm{Li}_{6+x} \mathrm{P}_{1-x} M_{x} \mathrm{~S}_{5} \mathrm{I}(M=\mathrm{Si}, \mathrm{Ge}, \mathrm{Sn})$, Chem. Mater. 31, 4936 (2019).

[87] A. Annamareddy and J. Eapen, Low dimensional stringlike relaxation underpins superionic conduction in fluorites and related structures, Sci. Rep. 7 (2017).

[88] C. R. A. Catlow, Atomistic mechanisms of ionic transport in fast-ion conductors, Faraday Trans. 86, 1167 (1990).

[89] C. R. A. Catlow, Defect processes and migration mechanisms in solid-state ionics, in Mat Sci Eng B-Solid (1992) pp. 375-382.

[90] C. E. Mohn and M. Krynski, Collective diffusion within the superionic regime of $\mathrm{Bi}_{2} \mathrm{O}_{3}$, Phys. Rev. B 101 (2020).

[91] M. Salanne, D. Marrocchelli, and G. W. Watson, Cooperative mechanism for the diffusion of $\mathrm{Li}^{+}$ions in $\mathrm{LiMgSO}_{4} \mathrm{~F}$, J. Phys. Chem. C 116, 18618 (2012).

[92] B. Zhang, L. Yang, L.-W. Wang, and F. Pan, Cooperative transport enabling fast Li-ion diffusion in thioLISICON $\mathrm{Li}_{10} \mathrm{SiP}_{2} \mathrm{~S}_{12}$ solid electrolyte, Nano Energy 62, 844 (2019).

[93] H. Zhang, X. Wang, A. Chremos, and J. F. Douglas, Superionic $\mathrm{UO}_{2}$ : A model anharmonic crystalline material, J. Chem. Phys. 150, 174506 (2019).

[94] X. He, Y. Zhu, and Y. Mo, Origin of fast ion diffusion in super-ionic conductors, Nature Comm. 8, 15893 (2017).

[95] M. Xu, J. Ding, and E. Ma, One-dimensional stringlike cooperative migration of lithium ions in an ultrafast ionic conductor, Appl. Phys. Lett. 101, 031901 (2012).

[96] I. Yokota, On the deviation from the Einstein relation observed for diffusion of $\mathrm{Ag}$ ions in $\alpha-\mathrm{Ag}_{2} \mathrm{~S}$ and others, J. Phys. Soc. Japan 21, 420 (1966).

[97] M. A. Zendejas and J. O. Thomas, Conduction mechanisms in solid electrolytes: $\mathrm{Na}^{+}$beta-alumina, Physica Scripta T33, 235 (1990). 
[98] C. Donati, J. F. Douglas, W. Kob, S. J. Plimpton, P. H. Poole, and S. C. Glotzer, Stringlike cooperative motion in a supercooled liquid, Phys. Rev. Lett. 80, 2338 (1998).

[99] B. J. Morgan and P. A. Madden, Relationships between atomic diffusion mechanisms and ensemble transport coefficients in crystalline polymorphs, Phys. Rev. Lett. 112, 145901 (2014).

[100] S. C. Glotzer, Spatially heterogeneous dynamics in liquids: Insights from simulation, Journal of NonCrystalline Solids 274, 342 (2000).

[101] H. Zhang, C. Zhong, J. F. Douglas, X. Wang, Q. Cao, D. Zhang, and J.-Z. Jiang, Role of string-like collective atomic motion on diffusion and structural relaxation in glass forming $\mathrm{Cu}-\mathrm{Zr}$ alloys, J. Chem. Phys. 142, 164506 (2015).

[102] Y.-J. Wang, J.-P. Du, S. Shinzato, L.-H. Dai, and S. Ogata, A free energy landscape perspective on the nature of collective diffusion in amorphous solids, Acta Mater. 157, 165 (2018).

[103] Z. Zhang, J. Zhang, H. Jia, L. Peng, T. An, and J. Xie, Enhancing ionic conductivity of solid electrolyte by lithium substitution in halogenated Li-argyrodite, J. Power Sources 450, 227601 (2020).

[104] C. Yu, Y. Li, M. Willans, Y. Zhao, K. R. Adair, F. Zhao, W. Li, S. Deng, J. Liang, M. N. Banis, R. Li, H. Huang, L. Zhang, R. Yang, S. Lu, Y. Huang, and X. Sun, Superionic conductivity in lithium argyrodite solid-state electrolyte by controlled Cl-doping, Nano Energy 69, 104396 (2020).

[105] B. Kozinsky, Transport in frustrated and disordered solid electrolytes, in Handbook of Materials Modeling (Springer International Publishing, 2018) pp. 1-20.

[106] W. Hayes, Superionic conductors, Contemp. Phys. 19, 469 (1978).

[107] M. Villa and J. L. Bjorkstam, The role of disorder in superionic conductors, Sol. Stat. Ionics. 1, 481 (1980).

[108] D. A. Keen, Disordering phenomena in superionic conductors, Journal of Physics: Condensed Matter 14, R819 (2002).

[109] S. Hull, Superionics: Crystal structures and conduction processes, Rep. Prog. Phys. 67, 1233 (2004).

[110] G. K. P. Dathar, J. Balachandran, P. R. C. Kent, A. J. Rondinone, and P. Ganesh, Li-ion site disorder driven superionic conductivity in solid electrolytes: a firstprinciples investigation of $\beta-\mathrm{Li}_{3} \mathrm{PS}_{4}$, J. Mater. Chem. A 5, 1153 (2017).

[111] D. Di Stefano, A. Miglio, K. Robeyns, Y. Filinchuk, M. Lechartier, A. Senyshyn, H. Ishida, S. Spannen- berger, D. Prutsch, S. Lunghammer, D. Rettenwander, M. Wilkening, B. Roling, Y. Kato, and G. Hautier, Superionic diffusion through frustrated energy landscape, Chem 5, 2450 (2019).

[112] K. E. Kweon, J. B. Varley, P. Shea, N. Adelstein, P. Mehta, T. W. Heo, T. J. Udovic, V. Stavila, and B. C. Wood, Structural, chemical, and dynamical frustration: Origins of superionic conductivity in closo-borate solid electrolytes, Chem. Mater. 29, 9142 (2017).

[113] M. Jorgensen, P. T. Shea, A. W. Tomich, J. B. Varley, M. Bercx, S. Lovera, R. Černý, W. Zhou, T. J. Udovic, V. Lavallo, T. R. Jensen, B. C. Wood, and V. Stavila, Understanding superionic conductivity in lithium and sodium salts of weakly coordinating closohexahalocarbaborate anions, Chem. Mater. 32, 1475 (2020).

[114] B. T. Leube, K. K. Inglis, E. J. Carrington, P. M. Sharp, J. F. Shin, A. R. Neale, T. D. Manning, M. J. Pitcher, L. J. Hardwick, M. S. Dyer, F. Blanc, J. B. Claridge, and M. J. Rosseinsky, Lithium transport in $\mathrm{Li}_{4.4} M_{0.4} M_{0.6}^{\prime} \mathrm{S}_{4}\left(M=\mathrm{Al}^{3+}, \mathrm{Ga}^{3+}\right.$, and $M^{\prime}=\mathrm{Ge}^{4+}$, $\left.\mathrm{Sn}^{4+}\right)$ : Combined crystallographic, conductivity, solid state NMR, and computational studies, Chem. Mater. 30, 7183 (2018).

[115] A. Düvel, P. Heitjans, P. Fedorov, G. Scholz, G. Cibin, A. V. Chadwick, D. M. Pickup, S. Ramos, L. W. L. Sayle, E. K. L. Sayle, T. X. T. Sayle, and D. C. Sayle, Is geometric frustration-induced disorder a recipe for high ionic conductivity?, J. Amer. Chem. Soc. 139, 5842 (2017).

[116] S. Breuer and M. Wilkening, Mismatch in cation size causes rapid anion dynamics in solid electrolytes: the role of the arrhenius pre-factor, Dalton Trans. 47, 4105 (2018).

[117] Y. Deng, C. Eames, B. Fleutot, R. David, J.-N. Chotard, E. Suard, C. Masquelier, and M. S. Islam, Enhancing the lithium ion conductivity in lithium superionic conductor LISICON solid electrolytes through a mixed polyanion effect, ACS Appl. Mater. Int. 9, 7050 (2017).

[118] M. A. T. Marple, B. G. Aitken, S. Kim, and S. Sen, Observation of a phonon softening effect on $\mathrm{Li}$ ion conduction in mixed-anion chalcogenide glasses, Chem. Mater. 30, 5896 (2018).

[119] Y. Zhang, Y. Zhao, and C. Chen, Ab initio study of the stabilities of and mechanism of superionic transport in lithium-rich antiperovskites, Phys. Rev. B 87 (2013).

[120] J. C. Dyre, P. Maass, B. Roling, and D. L. Sidebottom, Fundamental questions relating to ion conduction in disordered solids, Rep. Prog. Phys. 72, 046501 (2009). 